\title{
THE TRANSVERSE AND ROTATIONAL MOTIONS OF MAGNETOHYDRODYNAMIC KINK WAVES IN THE SOLAR ATMOSPHERE
}

\author{
M. Goossens ${ }^{1}$, R. Soler ${ }^{2}$, J. Terradas ${ }^{2}$, T. Van Doorsselaere ${ }^{1}$, And G. Verth ${ }^{3}$ \\ ${ }^{1}$ Centre for Mathematical Plasma Astrophysics, Katholieke Universiteit Leuven, Celestijnenlaan 200B, \\ bus 2400, B-3001 Herverlee, Belgium; marcel.goossens@ wis.kuleuven.be \\ 2 Departament de Física, Universitat de les Illes Balears, E-07122 Palma de Mallorca, Spain \\ ${ }^{3}$ Solar Physics and Space Plasma Research Centre (SP $\left.{ }^{2} \mathrm{RC}\right)$, The University of Sheffield, Hicks Building, Hounsfield Road, Sheffield S3 7RH, UK \\ Received 2014 March 12; accepted 2014 April 14; published 2014 May 16
}

\begin{abstract}
Magnetohydrodynamic (MHD) kink waves have now been observed to be ubiquitous throughout the solar atmosphere. With modern instruments, they have now been detected in the chromosphere, interface region, and corona. The key purpose of this paper is to show that kink waves do not only involve purely transverse motions of solar magnetic flux tubes, but the velocity field is a spatially and temporally varying sum of both transverse and rotational motion. Taking this fact into account is particularly important for the accurate interpretation of varying Doppler velocity profiles across oscillating structures such as spicules. It has now been shown that, as well as bulk transverse motions, spicules have omnipresent rotational motions. Here we emphasize that caution should be used before interpreting the particular MHD wave mode/s responsible for these rotational motions. The rotational motions are not necessarily signatures of the classic axisymmetric torsional Alfvén wave alone, because kink motion itself can also contribute substantially to varying Doppler velocity profiles observed across these structures. In this paper, the displacement field of the kink wave is demonstrated to be a sum of its transverse and rotational components, both for a flux tube with a discontinuous density profile at its boundary, and one with a more realistic density continuum between the internal and external plasma. Furthermore, the Doppler velocity profile of the kink wave is forward modeled to demonstrate that, depending on the line of sight, it can either be quite distinct or very similar to that expected from a torsional Alfvén wave.
\end{abstract}

Key words: magnetohydrodynamics (MHD) - Sun: chromosphere - Sun: corona - Sun: oscillations

Online-only material: animation, color figures

\section{INTRODUCTION}

The aim of this paper is to show that the motion of a magnetic flux tube due to a magnetohydrodynamic (MHD) kink wave is the sum of a spatially constant transverse and a nonaxisymmetric rotational motion. Thus far in the corona, Extreme Ultra-Violet (EUV) imagers such as the Transition Region and Coronal Explorer and the Atmospheric Imaging Assembly on board the Solar Dynamics Observatory have only permitted us to observe the bulk transverse motions associated with kink waves in thin coronal structures which have a greater EUV intensity relative to the background plasma, e.g., coronal loops. Although thus far they have not been identified directly in the corona, the kink wave's rotational motions could certainly contribute to the observed non-thermal line widths (e.g., McIntosh \& De Pontieu 2012). So far, the identification of these rotational motions in the corona via Doppler shift using spectrometers has been hampered by limited EUV spatial resolution. However, in cooler chromospheric spectral lines, e.g., $\mathrm{H} \alpha$ and $\mathrm{Ca}$ II $\mathrm{H}$, kink waves propagating along spicules/fibrils/mottels can be observed with much finer resolution from both ground-based telescopes and space-borne instruments (e.g., De Pontieu et al. 2012; Morton et al. 2012, 2013, 2014; Kuridze et al. 2012, 2013). Note also, in these cooler lines, omnipresent kink waves have been discovered in solar prominences (e.g., Lin et al. 2009). Using both the TRIPort Polarimetric Echelle-Littrow spectrograph (TRIPPEL) and the CRisp Imaging SpectroPolarimeter (CRISP) based at the Swedish Solar Telescope (SST), De Pontieu et al. (2012) have now discovered Doppler signals of ubiquitous rotational motions occurring in these thin, dense chromospheric structures, which are concurrent with transverse kink motions. De Pontieu et al. (2012) suggested that the rotational motions are predominantly signatures of the $m=0$ torsional Alfvén wave.

Since spicules display such obvious transverse kink motion, their Doppler signals should also contain evidence of the $m=1$ rotational motions to which they are coupled. It is a key purpose of this paper to investigate what the actual Doppler signatures of these coupled transverse and rotational $m=1$ kink motions look like. This is particularly important since De Pontieu et al. (2012) have now actually managed to resolve varying trends in Doppler blue and red shift normal to the axes of spicules in which ubiquitous kink waves are propagating. This means that for the first time we will not only be able to analyze the propagation of kink waves purely from their bulk transverse motion (as is usually done for kink waves observed in poorer resolution EUV lines in the corona), but we will also be able to observe this highly Alfvénic MHD wave in finer detail to see how the coupling between the transverse and rotational components develop with height in the lower solar atmosphere. This is especially timely since there is now evidence that the damping length for propagating kink waves in the lower atmosphere (less than heights of $15-20 \mathrm{Mm}$ ) is only about $10 \%$ of estimated coronal loop damping lengths (Morton et al. 2014). Mode conversion through the process of resonant absorption could play a dominant role in this, see, e.g., Terradas et al. (2010), Verth et al. (2010) and review by Goossens et al. (2011). To fully understand the dynamics, mode coupling, conversion, and damping of MHD waves in the chromosphere, detailed comparison of the transverse and rotational velocity components with height is crucial. 
This would lead to a great advance in understanding the structure and dynamics of, e.g., spicules, fibrils, and mottles. It is hoped that the current work will lead to more accurate interpretations of Doppler signal trends being resolved across these thin, dense, magnetic structures by these and other modern high resolution instruments, e.g., Interferometric BIdimensional Spectrometer (IBIS) at the Dunn Solar Telescope (DST), the Interface Region Imaging Spectrograph (IRIS), and the forthcoming Advanced Technology Solar Telescope (ATST).

To study the possible MHD wave modes that can propagate along thin, dense, chromospheric structures such as spicules, fibrils, and mottles, it is instructive to model these waveguides as axisymmetric magnetic cylinders. MHD wave modes which propagate along such structures are characterized by two wave numbers, the azimuthal wave number $m$, and the axial wave number $k_{z}$. In addition, particular modes can also have different nodes in the radial direction, and this number can be used to further classify the modes. For the particular type of MHD wave modes which have $m=1$, i.e., the kink mode, the wavelength is nearly always observed to be much greater than the actual width of solar waveguide. In this regime, the fundamental radial mode is the only trapped kink mode and is therefore the mode most relevant to solar applications. Goossens et al. $(2009,2012)$ have shown that the fundamental radial mode can be considered as a surface Alfvén wave. The MHD kink wave has a phase velocity between the internal, $v_{A, i}$, and external $v_{A, e}$ Alfvén speeds and is the only mode that displaces the axis of the magnetic flux tube and the tube as a whole, which is why is it one of the easiest MHD wave modes to identify in observations.

A popular conception of a MHD kink wave is that it is a wave that displaces the magnetic flux tube as a whole in the transverse direction with no other motion involved. However, we shall show that this concept is only correct when considering the actual plasma motion inside a sufficiently thin homogeneous flux tube that has a piece-wise variation between the internal and external plasma density. In that configuration, inside the flux tube the radial component $\xi_{r}$ and the azimuthal component $\xi_{\varphi}$ have the same amplitude, and the azimuthal component $\xi_{\varphi}$ is a quarter of a period ahead of the radial component $\xi_{r}$. The result is then a transverse periodic displacement within the flux tube. However, even in such a simple configuration, the velocity field for the MHD kink wave outside of the tube is actually dipolar in character and not purely transverse at all. It is only purely transverse motion within the tube boundary.

The popular model with a piece-wise, constant density is instructive but has its clear limitations. It fails to describe the fundamental properties of MHD waves that are due to nonuniformity present in more realistic solar flux tube models. Examples are (1) the fast damping of kink waves with frequencies between the internal and external values of the Alfvén frequency because of resonant absorption (see, e.g., Goossens et al. 1992, 2002; Ruderman \& Roberts 2002; Van Doorsselaere et al. 2004); (2) the presence of vorticity in kink waves (Goossens et al. 2012); and (3) MHD waves with mixed properties (see, e.g., Goossens 2008; Goossens et al. 2002, 2009, 2011). The degree to which the classic properties are present in a given MHD wave depends on the particular background through which the wave travels. An MHD wave mode that is characterized by the same wave numbers has different properties for different backgrounds. The displacement and velocity fields of the fundamental radial mode of kink MHD waves are different for different equilibrium distributions of density. In particular, the displacement and velocity fields for a model with non-uniform density are different from those for a model with a piece-wise constant density. Hence, it can be anticipated that the motion of a non-uniform magnetic cylinder due to a kink wave will be more complicated than the simple transverse motion of a thin, magnetic cylinder with a piece-wise constant density.

The paper is organized as follows. In the following section we recapitulate the equations for linear motions superimposed on a straight, axisymmetric magnetic cylinder. Section 3 considers what we mean by the thin flux tube approximation in relation to the kink mode wave variables, and Section 4 shows the displacement field for a thin tube (TT) with a density discontinuity at the boundary. In Section 5, we demonstrate how the kink wave displacement field is the sum of both transverse and rotational motion, and in Section 6, we consider the consequences of this for interpreting the line-of-sight (LOS) Doppler velocity profiles across oscillating magnetic flux tubes observed in the solar atmosphere. This idea is developed further in Section 7, where the Doppler velocity profiles across such oscillating flux tubes are forward modeled by integrating intensity along the LOS. In Section 8, we investigate the time/space evolution of the transverse and rotational components of the kink wave displacement field in flux tubes that have a continuous variation in density between their interior and exterior. In relation to this more realistic plasma configuration, the idea of a "quasi-mode" is discussed and the linearized, ideal MHD equations are solved numerically to provide insight into the dynamic process of mode conversion that naturally occurs in the presence of a resonant layer in such a flux tube. This physical process, known as resonant absorption, channels the kink wave energy from its transverse motion into predominantly non-axisymmetric rotational motion and therefore has important consequences for interpreting observed Doppler velocity profiles across solar magnetic waveguides.

\section{MHD KINK WAVES IN A MAGNETIC CYLINDER}

Our interest is in the motion of an axisymmetric magnetic cylinder with a straight magnetic field due to the fundamental radial mode of kink waves:

$$
\boldsymbol{B}_{\mathbf{0}}=B_{0} \mathbf{1}_{z} .
$$

In order to determine this motion, we need expressions for components of the Lagrangian displacement $\xi$ and the frequency of the wave $\omega$. The background model is independent of the spatial coordinates $\varphi, z$, and time, $t$. However, it is variable in the radial direction. Hence, the perturbed quantities are put proportional to

$$
\exp \left(i\left(m \varphi+k_{z} z-\omega t\right)\right)
$$

In Equation (2), $m$ is the azimuthal wave number, $k_{z}$ is the longitudinal wave number, and $\omega$ is the frequency. Since the background is variable in the radial direction, there is not a constant radial wave number. If we insist, we can use the number of nodes in the radial part of the eigenfunctions to distinguish between radial fundamental and overtone modes. Since our primary concern is studying the MHD kink mode on magnetically dominated solar atmospheric flux tubes, we treat the plasma as pressureless so that $v_{S}^{2}=0$ with $v_{S}$ the local speed of sound. The equations for linear MHD waves on a one-dimensional, pressureless cylinder with a straight field can be obtained from the more general equations by, e.g., Appert et al. (1974), Sakurai et al. (1991), Goossens 
et al. (1992), and Goossens et al. (1995) by putting the local speed of sound $v_{S}$ and the azimuthal component of the equilibrium magnetic field $B_{\varphi}$ equal to 0 . The resulting equations are given by, e.g., Goossens et al. (2009) and Goossens (2008). The solutions for an equilibrium with a piece-wise constant density can be given in terms of Bessel functions $J_{m}(x)\left(x=k_{i} r\right)$ in the internal part of the flux tube and $K_{m}(y)\left(y=k_{e} r\right)$ in the exterior region (see, e.g., Wentzel 1979; Wilson 1979, 1980; Spruit 1982; Edwin \& Roberts 1983). The internal and external radial wavenumbers $k_{i}$ and $k_{e}$, respectively, are defined as

$$
k_{i}^{2}=\Gamma_{i}\left(\omega^{2}\right)=\frac{\omega^{2}-\omega_{A i}^{2}}{v_{A, i}^{2}}, \quad k_{e}^{2}=-\Gamma_{e}\left(\omega^{2}\right)=-\frac{\omega^{2}-\omega_{A e}^{2}}{v_{A, e}^{2}} .
$$

The solutions are

$$
\begin{aligned}
& \xi_{\varphi, i}(r)=\alpha \frac{i m / r}{\rho_{i}\left(\omega^{2}-\omega_{A i}^{2}\right)} J_{m}(x), \\
& \xi_{r, i}(r)=\alpha \frac{k_{i}}{\rho_{i}\left(\omega^{2}-\omega_{A i}^{2}\right)} J_{m}^{\prime}(x), \\
& \xi_{\varphi, e}(r)=\beta \frac{i m / r}{\rho_{e}\left(\omega^{2}-\omega_{A e}^{2}\right)} K_{m}(y), \\
& \xi_{r, e}(r)=\beta \frac{k_{e}}{\rho_{e}\left(\omega^{2}-\omega_{A e}^{2}\right)} K_{m}^{\prime}(y),
\end{aligned}
$$

where $x=k_{i} r$ and $y=k_{e} r$. An accent denotes a derivative with respect to the argument $x$ or $y . \alpha$ and $\beta$ are constants. Continuity of total pressure and the radial component of the Lagrangian displacement leads to the dispersion relation

$$
F \frac{J_{m}^{\prime}\left(x_{0}\right) K_{m}\left(y_{0}\right)}{J_{m}\left(x_{0}\right) K_{m}^{\prime}\left(y_{0}\right)}=1
$$

with the quantity $F$ given by

$$
F=\frac{k_{i}}{k_{e}} \frac{\rho_{e}\left(\omega^{2}-\omega_{A e}^{2}\right)}{\rho_{i}\left(\omega^{2}-\omega_{A i}^{2}\right)},
$$

where $x_{0}=k_{i} R$ and $y_{0}=k_{e} R$. The dispersion relation (5) can be solved numerically. This was done for real frequencies by, e.g., Wentzel (1979), Wilson (1979, 1980) and Edwin \& Roberts (1983), and for complex frequencies by, e.g., Spruit (1982) and Cally (1985)

As we shall see later, the nature of the motion of the flux tube depends on the relative importance of the components $\xi_{r}$ and $\xi_{\varphi}$. The ratio of the azimuthal component to the radial component of the Lagrangian displacement for kink waves with $m=1$ in the interior part $(0 \leqslant r \leqslant R)$ of the magnetic tube, is

$$
\frac{\xi_{\varphi}(r)}{\xi_{r}(r)}=i \frac{1}{x} \frac{J_{1}(x)}{J_{1}^{\prime}(x)}
$$

The factor $i$ means that $\xi_{\varphi}$ is a quarter of a period ahead of $\xi_{r}$. For the fundamental radial mode $0 \leqslant x \leqslant x_{0} \leqslant x_{D J 1,1}<x_{J 1,1}$ where $x_{D J 1,1}$ is the first zero of $J_{1}^{\prime}(x)$ and $x_{J 1,1}$ is the first zero of $J_{1}(x)$. In order to better understand the result in Equation (7), realize that the function

$$
\frac{1}{x} \frac{J_{1}(x)}{J_{1}^{\prime}(x)}
$$

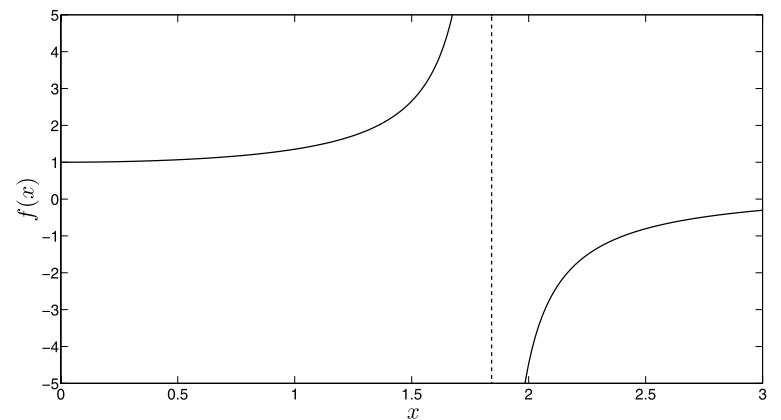

Figure 1. To illustrate the behavior of Equation (15), $f(x)$ is plotted against $x$ where $f(x)=J_{1}(x) /\left[x J_{1}^{\prime}(x)\right]$ and the asymptote shown by the vertical, dashed line at $x=x_{D J 1,1} \approx 1.84$, the first zero of $J_{1}^{\prime}(x)$.

is strictly increasing for $x \in\left[0, x_{D J 1,1}\right]$, and that for $x \in$ ]0, $x_{D J 1,1}[$ we have

$$
\begin{aligned}
& \frac{J_{1}(x)}{x J_{1}^{\prime}(x)}>1, \\
& \lim _{x \rightarrow 0} \frac{J_{1}(x)}{x J_{1}^{\prime}(x)}=1, \\
& \text { and } \lim _{x \rightarrow x_{D J 1,1}} \frac{J_{1}(x)}{x J_{1}^{\prime}(x)}=\infty .
\end{aligned}
$$

This means that the amplitude of the azimuthal component is always bigger than that of the radial component with the exception of $\lim _{x_{0} \rightarrow 0}$ which corresponds to the TT approximation. The function defined in Equation (8) is plotted in Figure 1.

Even for flux tubes with constant density, the amplitudes of the radial and azimuthal components are not equal. If we insist this to be the case in the whole flux tube, then we need to use the limit $R \rightarrow 0$.

\section{THIN TUBE APPROXIMATION}

The concept of the motion of a magnetic cylinder due to a kink wave to be a transverse motion finds its root in the displacement field pattern that emerges when the width of the tube is small compared to the wavelength. This regime is the socalled TT approximation. The Bessel functions $J_{m}(x)$ and $K_{m}(y)$ in Equation (5) are replaced with their first order asymptotic expansions. The dispersion relation (5) is reduced to

$$
1+F \frac{k_{e}}{k_{i}}=0
$$

The solution for the frequency is

$$
\omega^{2}=\frac{\rho_{i} \omega_{A i}^{2}+\rho_{e} \omega_{A e}^{2}}{\rho_{i}+\rho_{e}}=\frac{2}{\rho_{i}+\rho_{e}} k_{z}^{2} \frac{B_{0}^{2}}{\mu_{0}}=\omega_{k}^{2}
$$

and for the radial wave numbers $k_{i}$ and $k_{e}$,

$$
k_{i}^{2}=k_{e}^{2}=k_{z}^{2} \frac{\rho_{i}-\rho_{e}}{\rho_{i}+\rho_{e}}=k^{2} .
$$

In the TT approximation, the fundamental radial mode is the only trapped mode. The higher radial overtones in this regime are all leaky. This is why this fundamental mode is so relevant to observed solar atmospheric kink waves, where wavelengths are seen to be much larger than waveguide widths. The right-hand side of Equation (11) is almost invariably called the square of the 
kink frequency and denoted as $\omega_{k}^{2}$. In the TT approximation, the frequency is independent of the wave number, $m \geqslant 1$ as already noted in Goossens et al. (1992). Hence, all fluting modes with $m \geqslant 2$ have the same frequency as the kink mode with $m=1$. The radial wave numbers $k_{i}$ and $k_{e}$ depend, in a simple way, on the density contrast. As the density contrast decreases, the nature of the MHD wave becomes gradually more Alfvénic, as explained in Goossens et al. (2009). Since we are only interested in the kink mode, in what follows, we fix $m=1$.

The TT approximation to the eigenfunctions in the interior of the loop, i.e., $0 \leqslant r \leqslant R$, are

$$
\begin{aligned}
\frac{\xi_{r, i}(r)}{R} & =C, \\
\text { and } \frac{\xi_{\varphi, i}(r)}{R} & =i C .
\end{aligned}
$$

In the exterior part, i.e., $r \in[R, \infty[$, the TT eigenfunctions are

$$
\begin{aligned}
\frac{\xi_{r, e}(r)}{R} & =C(k R)^{2} \frac{d K_{1}(y)}{d y}, \\
\text { and } \frac{\xi_{\varphi, e}(r)}{R} & =-i C(k R) \frac{K_{1}(y)}{r / R} .
\end{aligned}
$$

Note that $\xi_{\varphi, e}(R)=-\xi_{\varphi, i}(R)=-i C$. As explained in Goossens et al. (2009), terms of order $\left(k_{z} R\right)^{2}$ and of higher order have been omitted when deriving Equation (13) unless the terms of order $\left(k_{z} R\right)^{2}$ are the first non-vanishing contribution to the expression under study. For example, the expressions for $\xi_{r, i}(r) / R$ and $\xi_{\varphi, i}(r) / R$ mean that these two quantities have equal amplitudes up to differences of order $\left(k_{z} R\right)^{2}$. The eigenfunctions are determined up to a multiplicative constant $C$, which can be used to specify, e.g., the radial displacement of the boundary of the flux tube. The radial and azimuthal components are $\pi / 2$ out of phase, but they have equal magnitudes and inside the flux tube they are constant. In the TT approximation, for $0 \leqslant r \leqslant R$, Equation (7) can be replaced with

$$
\frac{\xi_{\varphi}(r)}{\xi_{r}(r)}=i
$$

in agreement with Equation (9).

Apart from $\xi_{\varphi}$, the wave quantities are continuous at $r=R . \xi_{\varphi}$ varies in a discontinuous manner at $r=R$ with opposite values at the two sides of the interface. This discontinuous behavior is due to the change of sign of the factor $\omega^{2}-\omega_{A}^{2}$ when we move from the interior to the exterior of the loop. When the jump in $\omega_{A}$ is replaced by a continuous variation of $\omega_{A}$, new and valuable physics are introduced to the system. The local Alfvén frequency $\omega_{A}(r)$ is function of position $r$ and defines the Alfvén continuum. In addition to the Alfvén continuum modes that give rise to uncoordinated motions in time, the system supports a damped coordinated motion. Since complex eigenvalues cannot exist in ideal MHD, this coordinated motion is called a quasimode. This quasi-mode is often a remnant of an eigenmode that, for a uniform plasma system, has its eigenvalue in between the extremal values of the Alfvén frequency. The fundamental radial mode of kink waves on solar flux tubes is a well-known example of this situation.

Soler et al. (2013) have shown how to deal with the mathematical intricacies of quasi-modes of cylindrical flux tubes, and have given a general, analytical method for the computation of their eigenfunctions and complex frequencies (see earlier investigations for planar geometry by, e.g., Hollweg 1990). In the present paper, we shall use this method for computing eigenfunctions in Section 8.

For thin transitional layers, the behavior is only quasisingular, i.e., large but finite values are generated. For thicker transitional layers, there is not a hint of singularity or quasisingularity. Instead, what happens is that the displacement jumps logarithmically at the resonance position, with the jumps remaining finite. Physically, introducing a transitional layer with a continuous variation of $\omega_{A}$ causes damping of the MHD wave by resonant absorption. This process results in azimuthal components of displacement and magnetic field perturbation that are far larger in absolute value than the corresponding radial components in the non-uniform layer around the resonant position, i.e., $\xi_{\varphi}$ eventually overpowers $\xi_{r}$. How this process affects the time and space evolution of the transverse and rotational components of the kink wave displacement field is addressed in more detail in Section 8.

\section{TRANSVERSE MOTION OF A THIN MAGNETIC FLUX TUBE}

Equation (13) tells us that for $0 \leqslant r \leqslant R$

$$
\frac{\boldsymbol{\xi}(r)}{R}=\frac{\boldsymbol{\xi}_{i}(r)}{R}=C\left(\mathbf{1}_{r}+i \mathbf{1}_{\varphi}\right) .
$$

Let us recall that from Equation (2) to this point, we have suppressed the dependence on the spatial variables $\varphi, z$, and time $t$. Now is the time to reintroduce the dependence on the angle $\varphi$. Since $\operatorname{Re} \exp (i \varphi)=\cos (\varphi)$ and $i=\exp (i \pi / 2)$, we can use the following real representation of $\xi$ for $0 \leqslant r \leqslant R$,

$$
\frac{\boldsymbol{\xi}(r, \varphi)}{R}=\frac{\boldsymbol{\xi}_{i}(r, \varphi)}{R}=C\left(\cos \varphi \mathbf{1}_{r}-\sin \varphi \mathbf{1}_{\varphi}\right) .
$$

The interpretation of this motion is best done in a system of Cartesian coordinates with the $x y$ plane normal to the axis of the cylindrical magnetic tube that is the $z$ axis in our system of cylindrical coordinates $(r, \varphi, z)$. The unit vectors $\left(\mathbf{1}_{x}, \mathbf{1}_{y}\right)$ in the Cartesian system and the unit vectors in the polar system $\left(\mathbf{1}_{r}, \mathbf{1}_{\varphi}\right)$ are related by

$$
\begin{aligned}
& \mathbf{1}_{x}=\mathbf{1}_{r} \cos \varphi-\mathbf{1}_{\varphi} \sin \varphi \\
& \mathbf{1}_{y}=\mathbf{1}_{r} \sin \varphi+\mathbf{1}_{\varphi} \cos \varphi
\end{aligned}
$$

and

$$
\begin{aligned}
& \mathbf{1}_{r}=\mathbf{1}_{x} \cos \varphi+\mathbf{1}_{y} \sin \varphi \\
& \mathbf{1}_{\varphi}=-\mathbf{1}_{x} \sin \varphi+\mathbf{1}_{y} \cos \varphi .
\end{aligned}
$$

From here on, we drop the factor $1 / R$ in the expression for the Lagrangian displacement field $\xi$. In Cartesian coordinates, Equation (17) is

$$
\boldsymbol{\xi}=\left[\begin{array}{l}
\xi_{x} \\
\xi_{y}
\end{array}\right]=C\left[\begin{array}{l}
1 \\
0
\end{array}\right]=\boldsymbol{\xi}_{\mathrm{TR}}
$$

where $\boldsymbol{\xi}_{\mathrm{TR}}$ denotes classic transverse kink motion, where the entire movement of the internal plasma is unidirectional along the $x$ axis with constant amplitude. We have added the subscript 


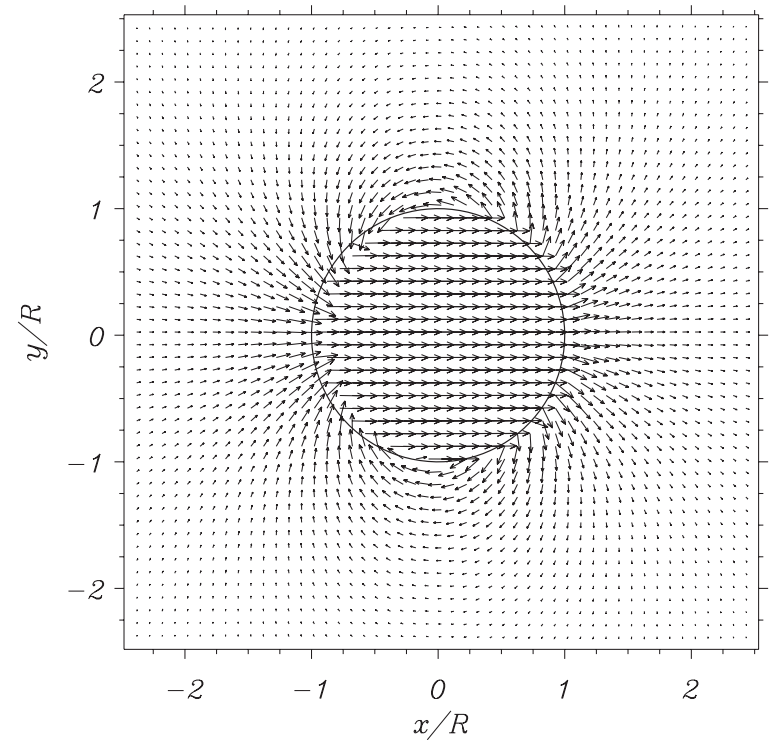

Figure 2. Kink wave ( $m=1)$ displacement field (arrows) in a piece-wise uniform tube. The circle represents the discontinuous boundary.

(TR) to emphasize this property. However, this rather simple, uniform displacement field is only true of a TT which has constant density values inside and outside the flux tube, and a discontinuity in these values at the boundary. This elementary result relies on the fact that inside the flux tube the radial and the azimuthal components have equal amplitudes, and that the azimuthal component is a quarter of a period ahead of the radial component. To reiterate, this situation only occurs for a magnetic flux tube that is uniform, has a piece-wise constant density, and is sufficiently thin. Any deviation of one of these three conditions results in a more complicated behavior than a pure transverse motion. The question is: how does the motion look when the amplitudes are different, and in particular, when the amplitude of the azimuthal component is larger than that of the radial component? Note also that the motion of the exterior plasma, even in the TT approximation, is not a pure, transverse motion as can be seen from the displacement vector field in Figure 2 .

In view of the discussion of the following section, we wish to point out the classic view of the transverse motion associated with the kink mode is a unidirectional motion that is independent of angle $\varphi$. The motion defined in Equation (22) has this particular property, which we label as $\boldsymbol{\xi}_{\mathrm{TR}}$.

\section{UNEQUAL AMPLITUDES, TRANSVERSE AND ROTATIONAL MOTIONS}

In this section, we consider the displacement field of an $m=1$ kink wave which has radial and azimuthal components with different amplitudes. It turns out that the interpretation of this motion is rather straightforward if we are familiar with its Cartesian representation. We shall focus on the dependence of the displacement on the angle $\varphi$. In view of what follows, it is instructive to first consider the simple case of the displacement field of an $m=0$ torsional Alfvén wave, i.e.,

$$
\boldsymbol{\xi}_{\mathrm{TAW}}=C \mathbf{1}_{\varphi} .
$$

In Cartesian coordinates this is equivalent to

$$
\boldsymbol{\xi}_{\mathrm{TAW}}=\left[\begin{array}{c}
\xi_{x} \\
\xi_{y}
\end{array}\right]=C\left[\begin{array}{l}
-\sin \varphi \\
+\cos \varphi
\end{array}\right]
$$
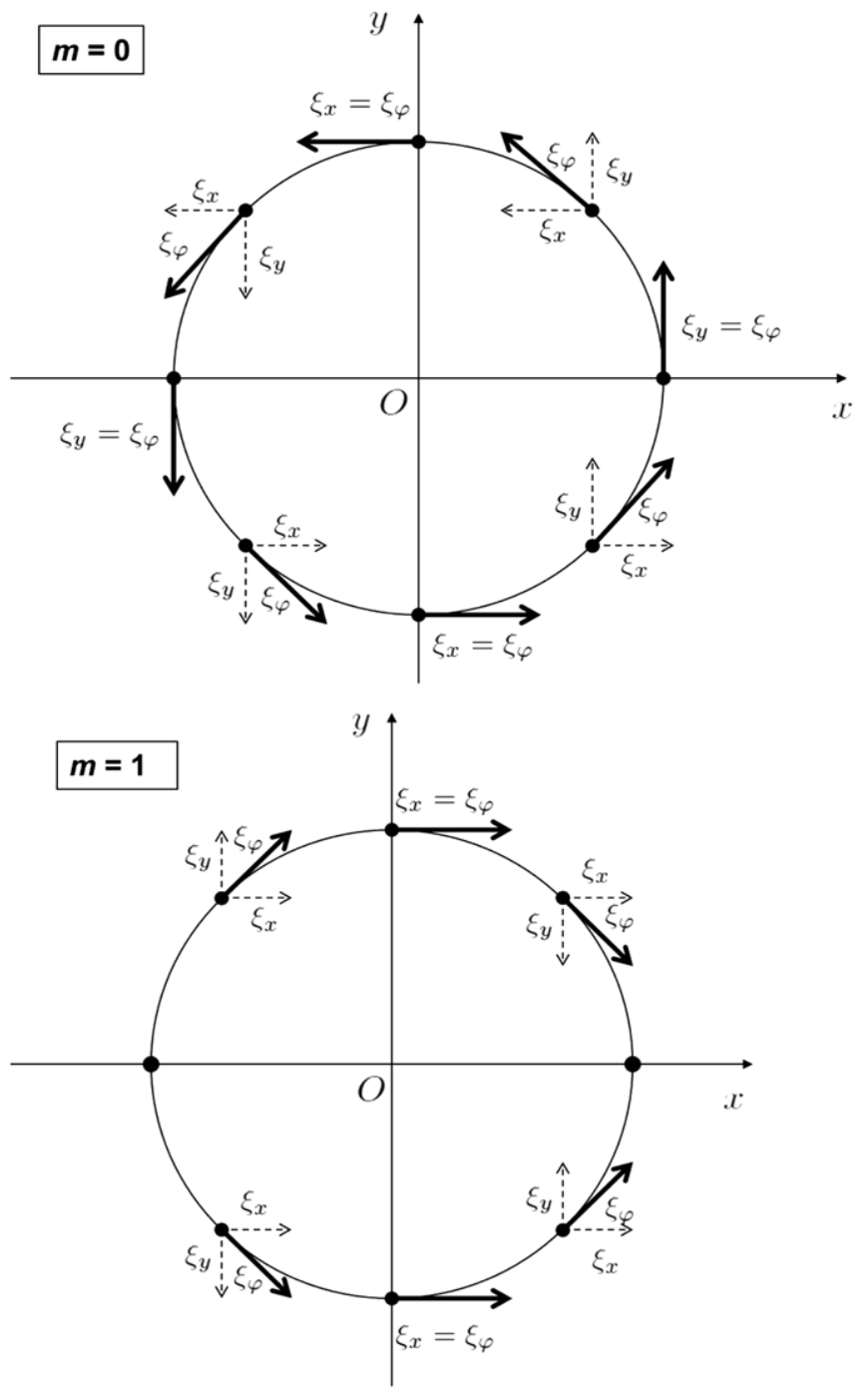

Figure 3. Top panel: from Equation (23), the $\boldsymbol{\xi}_{\mathrm{TAW}}$ displacement field an $m=0$ axisymmetric (or torsional) Alfvén wave is shown by bold arrows on a constant magnetic surface. From Equation (24), the relation between the polar $\xi_{\varphi}$ component and the Cartesian $\xi_{x}$ and $\xi_{y}$ components is also shown. Bottom panel: from Equation (25), the azimuthal component of the $m=1$ non-axisymmetric kink wave, denoted $\xi_{\mathrm{KAZ}}$, is shown by bold arrows on a constant magnetic surface. From Equation (26), the relation between the polar $\xi_{\varphi}$ component and the Cartesian $\xi_{x}$ and $\xi_{y}$ components is also shown.

The subscript $T$ refers to torsional and $A W$ to Alfvén wave. The displacement field of an axisymmetric or torsional Alfvén wave is shown on the top panel of Figure 3.

Let us now turn to the azimuthal component of the displacement field of a kink wave. Recall that kink refers to a nonaxisymmetric motion with $m=1$. The phase relation between the azimuthal and radial components in the previous section was determined by $\xi_{\varphi} / \xi_{r}=i$. Since $\operatorname{Re}(i \exp (i \varphi))=-\sin \varphi$, we choose as representation of the kink azimuthal motion

$$
\boldsymbol{\xi}_{\mathrm{KAZ}}=-C_{\mathrm{AZ}} \sin \varphi \mathbf{1}_{\varphi} .
$$

Here the subscript $\mathrm{K}$ refers to kink $(m=1)$ and AZ to the azimuthal component. In Cartesian coordinates,

$$
\xi_{\mathrm{KAZ}}=\left[\begin{array}{l}
\xi_{x} \\
\xi_{y}
\end{array}\right]=C_{\mathrm{AZ}} \sin \varphi\left[\begin{array}{c}
\sin \varphi \\
-\cos \varphi
\end{array}\right] .
$$

The $\boldsymbol{\xi}_{\mathrm{KAZ}}$ azimuthal displacement field of the $m=1 \mathrm{kink}$ wave is shown at a constant magnetic surface in bottom 
panel of Figure 3. From Equation (26), it is clear that the azimuthal displacement has a transverse component. However, this transverse component is a function of the angle $\varphi$. It differs from the classic transverse motion associated with a kink mode that is independent of the angle $\varphi$.

Let us now consider a displacement field with radial and azimuthal components with different amplitudes, respectively, $C_{\mathrm{TR}}$ and $C_{\mathrm{AZ}} \cdot C_{\mathrm{TR}}$ and $C_{\mathrm{AZ}}$ are both positive, and $C_{\mathrm{TR}} \leqslant C_{\mathrm{AZ}}$. This is a step toward understanding what happens in a nonuniform plasma cylinder. Note that the amplitudes $C_{\mathrm{TR}}$ and $C_{\mathrm{AZ}}$ depend on position in a non-uniform plasma.

The displacement field in polar coordinates is

$$
\boldsymbol{\xi}=C_{\mathrm{TR}} \cos \varphi \mathbf{1}_{r}-C_{\mathrm{AZ}} \sin \varphi \mathbf{1}_{\varphi} .
$$

$C_{\mathrm{TR}}$ and $C_{\mathrm{AZ}}$ are the amplitudes of, respectively, the radial and azimuthal components.

The aim now is to rewrite (27) as the sum of spatially constant transverse motion and a remaining part that depends on the angle $\varphi$. The recipe to do that is given by Equation (17). Hence, we rewrite (27) as

$$
\begin{aligned}
\boldsymbol{\xi}= & C_{\mathrm{TR}}\left(\cos \varphi \mathbf{1}_{r}-\sin \varphi \mathbf{1}_{\varphi}\right) \\
& -\left(C_{\mathrm{AZ}}-C_{\mathrm{TR}}\right) \sin \varphi \mathbf{1}_{\varphi} \\
= & C_{\mathrm{TR}} \mathbf{1}_{x}-C_{\mathrm{ROT}} \sin \varphi \mathbf{1}_{\varphi},
\end{aligned}
$$

where

$$
C_{\mathrm{ROT}}=C_{\mathrm{AZ}}-C_{\mathrm{TR}}
$$

is the amplitude of the non-axisymmetric, rotational motion. The interpretation of (28) follows immediately from Equations (17), (22), and (25). The first term of the right hand side of Equation (28) is the unidirectional transverse motion with amplitude $C_{\mathrm{TR}}$, the second term is the non-axisymmetric kink rotational motion. For completeness, Equation (28) in Cartesian coordinates is equivalent to

$$
\xi=\left[\begin{array}{l}
\xi_{x} \\
\xi_{y}
\end{array}\right]=C_{\mathrm{TR}}\left[\begin{array}{l}
1 \\
0
\end{array}\right]-C_{\mathrm{ROT}} \sin \varphi\left[\begin{array}{c}
-\sin \varphi \\
\cos \varphi
\end{array}\right]
$$

Let us reiterate: the first term on the right-hand side of either of Equation (28) or Equation (30) is the classic, pure transverse motion expected for a TT with piece-wise constant density. It is spatially constant and represents a uniform translation of the whole flux tube. This is consistent with the common view of the transverse motion associated with a kink mode. The second term is a non-axisymmetric kink rotational motion. The relative importance of the two terms depends on how much bigger $C_{\mathrm{AZ}}$ is compared to $C_{\mathrm{TR}}$. In all its simplicity, Equation (30) is an important result. It shows that the motion is the sum of a pure transverse motion and a non-axisymmetric, rotational motion. This result could have been anticipated as Goossens et al. (2012) showed that kink waves do have vorticity.

Let us point out that the second term in Equation (30) also contains a transverse component. This transverse component, $C_{\text {ROT }} \sin ^{2} \varphi$, varies with the angle $\varphi$ as $\sin ^{2} \varphi$. For $C_{\text {ROT }}>0$ it enhances the classic, constant transverse motion. It is zero for $\varphi=0, \pi$ and maximal for $\varphi=\pi / 2,3 \pi / 2$. For $C_{\mathrm{ROT}}<0$ this transverse motion counteracts the spatially constant transverse motion, It can change the direction of the full transverse motion if $\left|C_{\mathrm{ROT}}\right|>C_{\mathrm{TR}}$. This change of direction occurs $\varphi=\arcsin \left(C_{\mathrm{TR}} /\left|C_{\mathrm{ROT}}\right|\right)$.

Note also that Equation (30) tells us that there is a component perpendicular to the constant transverse motion, i.e., $-C_{\mathrm{ROT}} \sin \varphi \cos \varphi$. This motion vanishes for $\varphi=$ $0, \pi / 2,3 \pi / 2, \pi$ and is extremal for $\varphi=\pi / 4$, where it is equal to $-C_{\text {ROT }} / 2$.

Equation (28) has simple applications. First, we apply it to the kink motion in the external plasma in the case of a thin flux tube, in order to better understand Figure 2. The relevant amplitudes are now a function of position. They follow from Equation (14) as follows:

$$
\begin{aligned}
C_{\mathrm{TR}} & =C(k R)^{2} \frac{d K_{1}(y)}{d y}, \\
C_{\mathrm{AZ}} & =-C(k R) \frac{K_{1}(y)}{r / R}, \\
\text { and } C_{\mathrm{ROT}} & =C_{\mathrm{AZ}}-C_{\mathrm{TR}} .
\end{aligned}
$$

On the boundary $r=R$ the amplitudes are

$$
C_{\mathrm{TR}}=C, \quad C_{\mathrm{AZ}}=-C, \quad C_{\mathrm{ROT}}=-2 C
$$

and the displacement is

$$
\boldsymbol{\xi}_{e}(R, \varphi)=C \mathbf{1}_{x}+2 C \sin \varphi \mathbf{1}_{\varphi}
$$

Let us use Equation (33) to illustrate our previous comments on the effect of the second term on the right hand side of Equation (30) on transverse and perpendicular motion. We rewrite Equation (33) as

$$
\boldsymbol{\xi}_{e}(R, \varphi)=\left(C-2 C \sin ^{2} \varphi\right) \mathbf{1}_{x}+2 C \sin \varphi \cos \varphi \mathbf{1}_{y} .
$$

The motion due to the second term on the right hand side of Equation (34) counteracts the classic constant motion. It changes the direction of total transverse motion when $\varphi=$ $\arcsin \sqrt{2} / 2=\pi / 4$. For $\varphi \in]-\pi / 4, \pi / 4[$, the total transverse motion is directed along the positive $x$ axis. For $\varphi \in] \pi / 4,3 \pi / 4[$, the total transverse motion is in the opposite direction. Note also that there is perpendicular motion,

$$
\xi_{y}=2 C \sin \varphi \cos \varphi,
$$

which is positive for $\varphi \in] 0, \pi / 2$ [ and negative for $\varphi \in]-\pi / 2,0[$. It is maximal for $\varphi= \pm \pi / 4$ and is equal to $\pm C$.

Equation (34) can also help us to understand dissipationless damping of transverse motions, if we use the following toy model. Starting with a pure transverse motion with $C_{\mathrm{TR}}=$ $C_{\mathrm{AZ}}=C$ and letting kinetic energy in the radial motions decay in time by a transfer of energy to azimuthal motions according to

$$
C_{\mathrm{TR}}=C \exp \left(-t / \tau_{D}\right)
$$

and believing that energy is conserved by adopting

$$
C_{\mathrm{TR}}^{2}+C_{\mathrm{AZ}}^{2}=2 C^{2}
$$

so that

$$
\left.C_{\mathrm{ROT}}=C\left(2-\exp \left(-2 t / \tau_{D}\right)\right)^{1 / 2}-\exp \left(-t / \tau_{D}\right)\right) .
$$

The motion changes from a purely transverse motion $\boldsymbol{\xi}_{\mathrm{TR}}$, as defined in Equation (22), to a purely rotational motion $\boldsymbol{\xi}_{\mathrm{KAZ}}$, as defined by Equation (26) with $C_{\mathrm{AZ}}$ replaced with $\sqrt{2} C$ if we adopt the simple rule for the conservation of energy. This is the dissipationless damping of transverse waves that operates in resonant absorption. We are aware that matters are more complicated with the wave variables depending on 

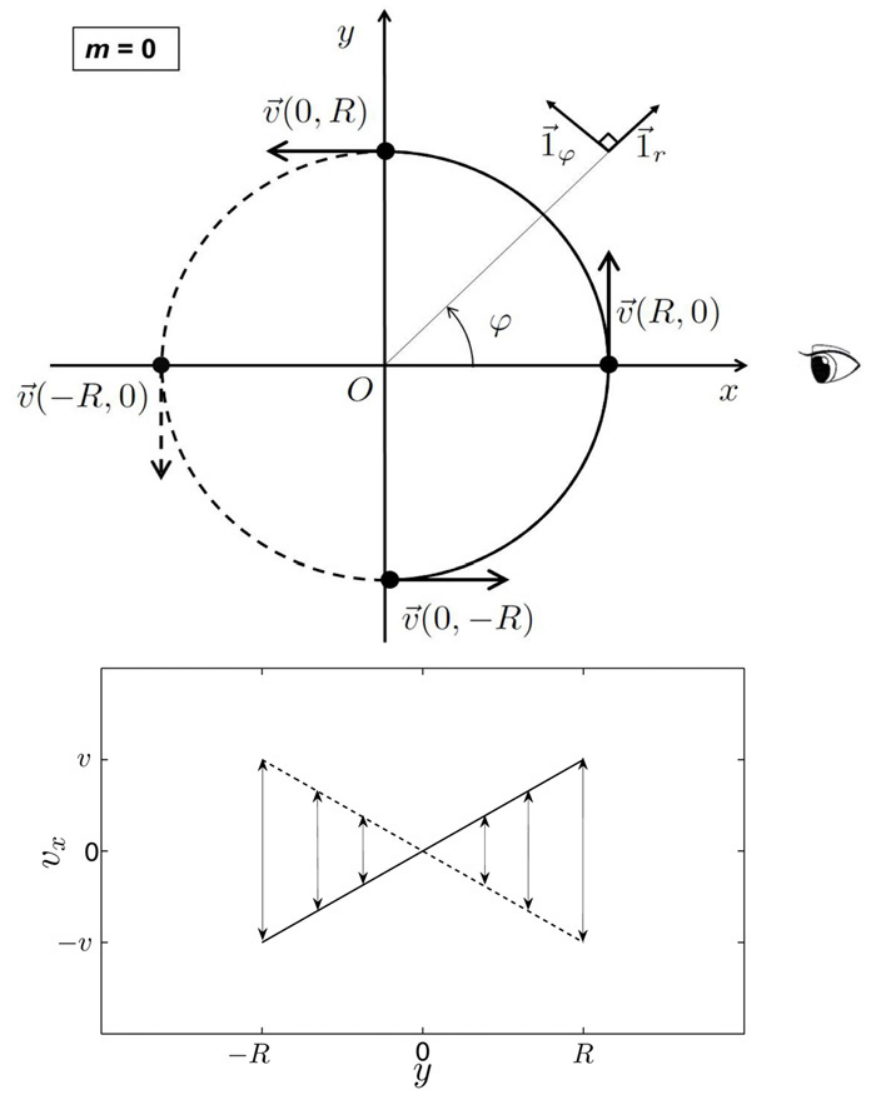

Figure 4. Top panel: in $(x, y)$ Cartesian coordinates, since the Doppler profile of the $m=0$ torsional Alfvén wave is independent of the LOS, this is chosen to be along the positive $x$ axis, perpendicular to a flux tube of radius $R$ oscillating with a torsional Alfvén wave $(m=0)$ of velocity amplitude $\boldsymbol{v}$ defined in Equation (38). The unit vectors for $\varphi$ and $r$ are also shown. Bottom panel: the extremal values of LOS Doppler velocity (the $v_{x}$ component of $\boldsymbol{v}$ ) shown by dashed and solid lines.

spatial position, and energy of the azimuthal motions being concentrated in a layer around the point of resonance as will be shown in more detail by the numerical, linearized MHD simulation described in Section 8. Nevertheless, this toy model captures the essence of the mechanism. The transverse motions undergo dissipationless damping because of a transfer of energy of radial motions to azimuthal motions.

\section{EFFECT OF UNEQUAL AMPLITUDES OF TRANSVERSE AND ROTATIONAL COMPONENTS ON DOPPLER VELOCITY PROFILES}

In Sections 2-5, we only considered the displacement vector $\xi$. In this section, we want to consider the possible Doppler velocity profiles across flux tubes oscillating with either $m=0$ torsional Alfvén waves or $m=1$ kink waves. Therefore, we introduce the velocity vector $\boldsymbol{v}$ which is simply proportional to $\xi$. As a natural continuation from the previous section, we want to show the effect of unequal amplitudes, transverse and rotational, on various LOS Doppler velocity profiles.

\subsection{Torsional Alfvén Wave}

Since there is no preferred direction in the horizontal plane ( $\boldsymbol{B}_{0}=B_{0} \mathbf{1}_{z}$ ) for viewing the $m=0$ torsional Alfvén wave, we can choose the direction toward the observer in the direction of

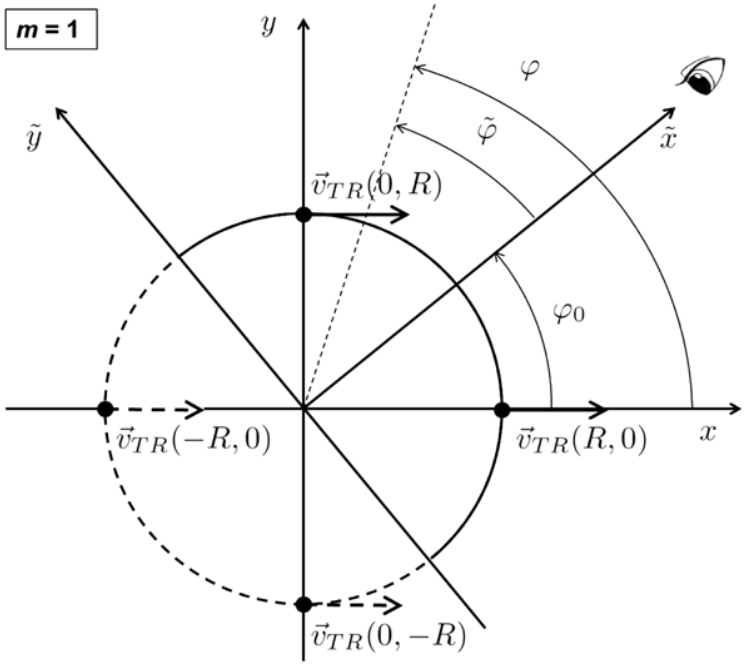

Figure 5. Here a flux tube is centered on the origin of $(x, y)$ Cartesian coordinates such that the classic kink transverse motion, $\boldsymbol{v}_{\mathrm{TR}}$ defined in Equation (22), is aligned with the $x$ axis. The angle measured anticlockwise from the positive $x$ axis is $\varphi$. An arbitrary LOS makes an angle $\varphi_{0}$ with the positive $x$ axis. A new Cartesian coordinate system, $(\tilde{x}, \tilde{y})$, is introduced such that the $\tilde{x}$ axis is aligned with the LOS. The angle relative to the positive $\tilde{x}$ axis is labeled as $\tilde{\varphi}$.

the $x$ axis (see top panel of Figure 4). Then we have that

$$
\boldsymbol{v}=v \mathbf{1}_{\varphi},
$$

where $v$ is the maximum velocity amplitude for the torsional Alfvén wave, and the $\varphi$ unit vector given in terms of the $x$ and $y$ Cartesian unit vectors is given by Equation (21). Then the LOS velocity component along the $x$ axis is simply

$$
v_{x}=\boldsymbol{v} \cdot \mathbf{1}_{x}=-v \sin \varphi .
$$

Now on the tube boundary $r=R$,

$$
\begin{aligned}
& x=R \cos \varphi, \\
& y=R \sin \varphi,
\end{aligned}
$$

and

$$
x^{2}=R^{2}-y^{2} .
$$

Insert Equation (41) in Equation (39) and find

$$
v_{x}=-v \frac{y}{R} \text { for }-R \leqslant y \leqslant R
$$

Equation (43) tells us how the Doppler velocity changes across the flux tube. Hence, for an observer, the variation of the Doppler velocity with respect to $y$ is linear, independent of the LOS (see bottom panel of Figure 4).

\subsection{Kink Wave}

Kink motions are non-axisymmetric and therefore the Doppler velocity profile is not independent of the LOS. In this section, we choose the classic kink transverse motion, $\boldsymbol{v}_{\mathrm{TR}}$, as defined in Equation (22), to be fixed along the direction of the $x$ axis and allow the LOS to vary with respect to this direction. We define a new coordinate system $(\tilde{x}, \tilde{y})$ such that the LOS is along the positive $\tilde{x}$ axis and the anticlockwise angle from the positive $x$ axis and $\tilde{x}$ axis is $\varphi_{0}$. We can now define a new angle $\tilde{\varphi}$ which is measured anti-clockwise from the positive $\tilde{x}$ axis (see 

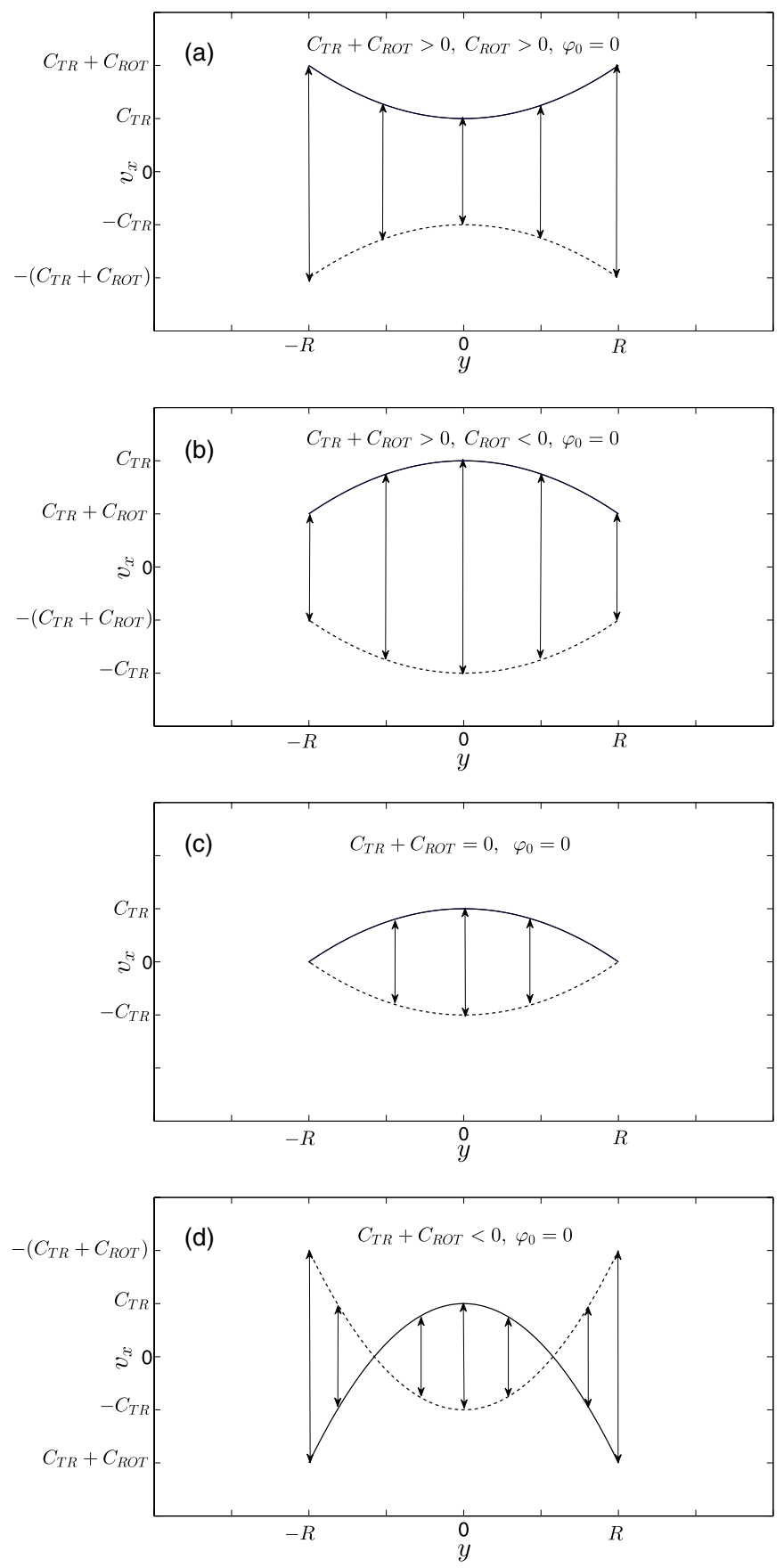

Figure 6. Choosing the LOS to be parallel to $\boldsymbol{v}_{\mathrm{TR}}$, i.e., $\varphi_{0}=0$, the LOS Doppler velocity component is given by $v_{x}$. Four distinct Doppler signatures are shown for various values of $C_{\mathrm{ROT}}$. In all cases $C_{\mathrm{TR}}>0$.

Figure 5). Hence, we have the following relations,

$$
\begin{gathered}
\varphi=\varphi_{0}+\tilde{\varphi}, \\
\tilde{x}=R \cos \tilde{\varphi}, \\
\tilde{y}=R \sin \tilde{\varphi},
\end{gathered}
$$

and

$$
\tilde{x}^{2}=R^{2}-\tilde{y}^{2} .
$$

\subsubsection{Special Case I $\left(\varphi_{0}=0\right)$}

When $\varphi_{0}=0$, the LOS is along the $x$ axis and aligned with the classic kink transverse motion. Hence, in

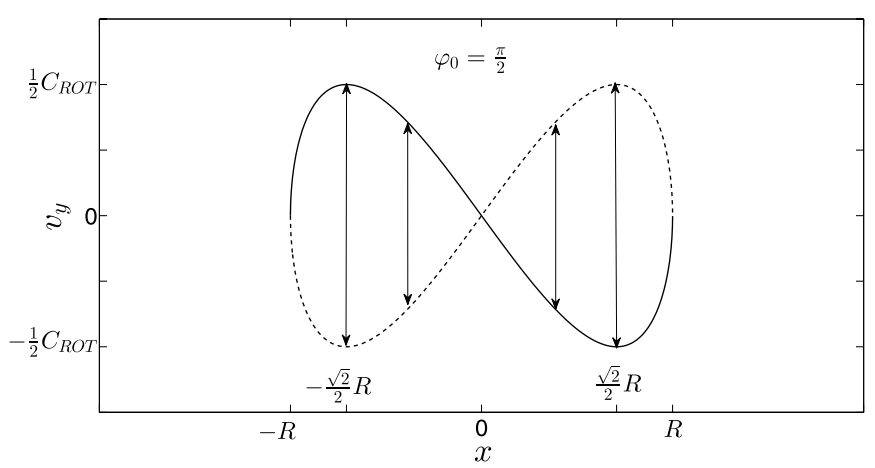

Figure 7. Choosing the LOS to be perpendicular to $\boldsymbol{v}_{\mathrm{TR}}$, i.e., $\varphi=\pi / 2$, the LOS Doppler velocity component is given by $v_{y}$. The Doppler signature is now independent of $C_{\mathrm{TR}}$ and only depends on $C_{\mathrm{ROT}}$.

Equations (44)-(47), $\tilde{\varphi}=\varphi, \tilde{x}=x$, and $\tilde{y}=y$. In this scenario, only the $v_{x}$ component contributes to the observed Doppler velocity signal, and this is governed by the following equation,

$$
v_{x}=C_{\mathrm{TR}}+C_{\mathrm{ROT}} \sin ^{2} \varphi .
$$

For this particular LOS, Equation (46) is equal to Equation (41). Using Equation (41), Equation (48) can be written as

$$
v_{x}=C_{\mathrm{TR}}+C_{\mathrm{ROT}}\left(\frac{y}{R}\right)^{2} .
$$

Equation (49) shows that the variation of the Doppler velocity across the flux tube is now controlled by a quadratic function of the coordinate $y$. Its graphical representation is a parabola. Depending on the sign and magnitude of $C_{\mathrm{ROT}}$ relative to $C_{\mathrm{TR}}$, this quadratic equation results in the four distinct parabolic Doppler velocity profiles shown in Figures 6(a)-(d). In Figure 6, we choose $C_{\mathrm{TR}}>0$ in all four cases. Note for $\varphi_{0}=0$, the Doppler velocity profile is always even about the tube center $(y=0)$. In Figure 6(a), $C_{\mathrm{ROT}}$ has the same sign as $C_{\mathrm{TR}}$, i.e., rotational and transverse motions are in phase. In Figures 6(b)-(d), $C_{\mathrm{ROT}}$ has the opposite sign as $C_{\mathrm{TR}}$, i.e., rotational and transverse motions are in anti-phase.

$$
\text { 6.2.2. Special Case II }\left(\varphi_{0}=\pi / 2\right)
$$

When $\varphi_{0}=\pi / 2$, the LOS is aligned with the $y$-axis and is therefore perpendicular to the classic kink transverse motion. In Equations (44)-(47) we have $\tilde{\varphi}=\varphi-\pi / 2, \tilde{x}=y$, and $\tilde{y}=-x$. From this particular LOS, the classic kink transverse motion does not contribute anything to the observed Doppler velocity. Only the $v_{y}$ component of rotational motion does this. This is governed by

$$
v_{y}=-C_{\mathrm{ROT}} \sin \varphi \cos \varphi .
$$

For this particular LOS, Equation (46) is equivalent to Equation (40), and Equation (47) takes the same form as Equation (42). Using Equations (40) and (42) in Equation (50) gives

$$
v_{y}=-C_{\mathrm{ROT}} \frac{x}{R}\left[1-\left(\frac{x}{R}\right)^{2}\right]^{1 / 2} .
$$

Hence, the Doppler velocity signal is zero at $x=0$ and $x= \pm R$. The extremal values are at $x= \pm R \sqrt{2} / 2$ and have magnitude $\left|C_{\mathrm{ROT}}\right| / 2$. Note that these extremal values are in anti-phase (see Figure 7). Hence, for $\varphi_{0}=\pi / 2$, the Doppler velocity profile is always odd about the tube center $(x=0)$. 
Note that this has a very important consequence in accurately interpreting the presence of torsional Alfvén waves or kink waves from observed Doppler velocity profiles. Comparing the Doppler velocity profile of the kink wave in Figure 7, between $x= \pm R \sqrt{2} / 2$, there is practically no difference in the Doppler signature of the torsional Alfvén wave shown in the lower panel of Figure 4. This ambiguity is further discussed in Section 7 where the LOS Doppler velocity is forward modeled, also taking into account integrated intensity.

\subsubsection{General Case}

For any LOS, i.e., an arbitrary value of $\varphi_{0}$, the observed Doppler velocity profile is governed by

$$
\begin{aligned}
v_{\tilde{x}} & =C_{\mathrm{TR}} \cos \varphi_{0} \\
& +C_{\mathrm{ROT}}\left(\sin ^{2} \varphi \cos \varphi_{0}-\sin \varphi \cos \varphi \sin \varphi_{0}\right) .
\end{aligned}
$$

We use Equations (44) and (46) and elementary trigonometric identities to rewrite Equation (52) in a form that describes the Doppler velocity amplitude from an arbitrary LOS in terms the LOS angle $\varphi_{0}$ relative to the positive $x$ axis and $y_{*}=\tilde{y} / R$ only, i.e.,

$$
\begin{aligned}
v_{\tilde{x}} & =\underbrace{C_{\mathrm{TR}} \cos \varphi_{0}}_{1 \mathrm{st} \text { term }} \\
& +C_{\mathrm{ROT}}[\underbrace{y_{*}^{2} \cos \varphi_{0}}_{\text {2nd term }}+\underbrace{y_{*}\left(1-y_{*}^{2}\right)^{1 / 2} \sin \varphi_{0}}_{3 \mathrm{rd} \text { term }}] .
\end{aligned}
$$

It can be seen that Equation (53) is a linear superposition of three distinct terms. Our toy model assumes fixed values of $\varphi_{0}, C_{\mathrm{TR}}$, and $C_{\mathrm{ROT}}$, hence the first term is simply a constant proportional to $C_{\mathrm{TR}}$. The first term represents the LOS Doppler velocity component of the classic kink transverse motion as defined in Equation (22). The second and third terms are both proportional to $C_{\mathrm{ROT}}$, and are even and odd functions about $y_{*}=0$, respectively. When the LOS is aligned with the classic kink transverse motion, i.e., $\varphi_{0}=0$ or $\varphi_{0}=\pi$, then the third term is zero and only the first and second terms contribute to the LOS Doppler velocity component, in agreement with Special case $I$ in Section 6.2.1. When the LOS is perpendicular to the classic kink transverse motion, i.e., $\varphi_{0}=\pi / 2$ or $\varphi_{0}=3 \pi / 2$, then the first and second terms are zero, and only the third term contributes to the LOS Doppler velocity component, in agreement with Special case II in Section 6.2.2. However, for an LOS that is neither aligned nor perpendicular to the classic kink transverse motion, all three terms are non-zero. The sum of the first and second terms produces a parabolic variation as discussed in Special case I in Section 6.2.1. The third term adds a variation, as discussed in Special case II in Section 6.2.2. Equation (53) clearly shows the continuous change in the Doppler velocity as function of $y$ as the LOS varies between the two extremes of being aligned with $\left(\varphi_{0}=0, \pi\right)$, and perpendicular to $(\varphi=\pi / 2,3 \pi / 2)$, the classic kink transverse motion. To illustrate the different possible types of Doppler velocity profiles in this scenario we choose $\varphi_{0}=\pi / 4$. In fact for $\varphi_{0}=\pi / 4, \cos \varphi_{0}=\sin \varphi_{0}=\sqrt{2} / 2$ and Equation (53) becomes

$$
v_{\tilde{x}}=\frac{\sqrt{2}}{2}\left\{C_{\mathrm{TR}}+C_{\mathrm{ROT}}\left[y_{*}^{2}+y_{*}\left(1-y_{*}^{2}\right)^{1 / 2}\right]\right\} .
$$
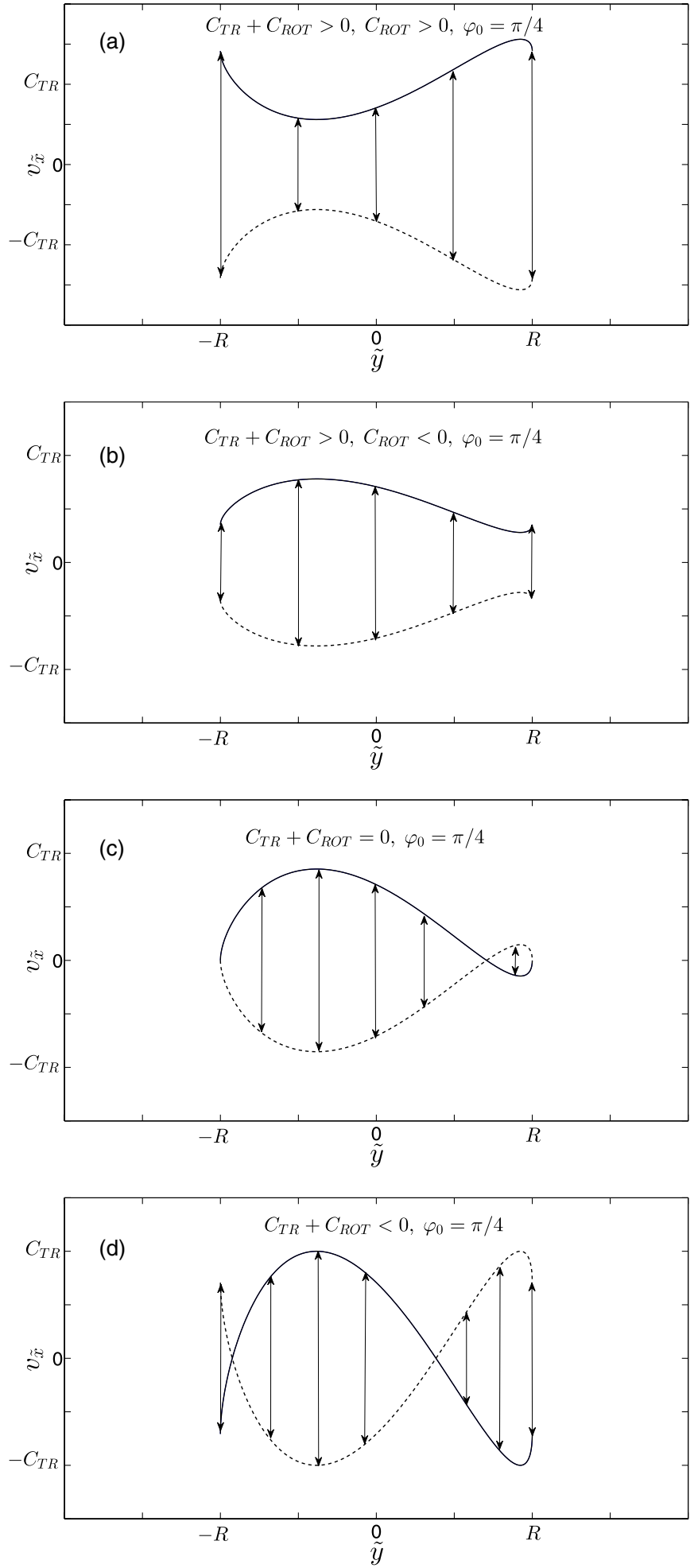

Figure 8. Choosing the LOS along $\varphi_{0}=\pi / 4$, the Doppler velocity is given by $v_{\tilde{x}}$ given in Equation (54). The combinations of $C_{\mathrm{TR}}$ and $C_{\mathrm{ROT}}$ are chosen to be the same as Figures 6(a)-(d) to illustrate how much the LOS Doppler velocity profile varies between $\varphi_{0}=0$ and $\varphi_{0}=\pi / 4$.

Equation (54) is plotted in Figures 8(a)-(d) for the same combinations of $C_{\mathrm{TR}}$ and $C_{\mathrm{ROT}}$ shown in Figures 6(a)-(d) to compare how different the Doppler velocity profiles look with a $\pi / 4$ difference between the viewing angles. Hence, the LOS angle relative to the direction of the classic transverse kink motion can have a crucial effect on accurately interpreting the 
Doppler velocity signatures of the kink wave. A forward model of Doppler velocity for both the torsional Alfvén and kink waves, taking into account both LOS and integrated intensity, is described in the next section.

\section{FORWARD MODEL FOR DOPPLER VELOCITY}

In this section, we study the observability as Doppler signatures of the torsional Alfvén and kink waves by integrating intensity and allowing the LOS to vary. To that end, we choose to model chromospheric spicules, in which ubiquitous transverse and rotational motions have been observed. As in the previous sections, we model such a spicule as a plasma cylinder. Since spicules are observed to be overdense relative to the ambient plasma, we fix $\rho_{i} / \rho_{e}=10$ (e.g., Beckers 1968). First, we only consider a piece-wise constant density profile. We take $10,000 \mathrm{~K}$ as the temperature for the system to mimic the formation temperature of the $\mathrm{Ca}$ II $\mathrm{H}$ line in which spicule transverse and rotational motion are clearly observed (e.g., He et al. 2009a, 2009b; Verth et al. 2011; Okamoto \& De Pontieu 2011; De Pontieu et al. 2012).

We numerically implement the velocity field given by Equations (4)-(5) on a grid, with a velocity amplitude of $20 \mathrm{~km} \mathrm{~s}^{-1}$. At each grid point, we then specify the emission as a Gaussian, with a spectral line width taken as the thermal velocity of the plasma (corresponding to calcium ions at the given temperature). The height of the Gaussian is taken proportional to the local density. This approximation is often made in estimating spicule plasma density from metallic line intensity, e.g., Makita (2003), Bjølseth (2008) and Okamoto \& De Pontieu (2011). The emission is then integrated along the LOS. The procedure used is based upon the work of Van Doorsselaere \& Nakariakov (2008) and similar to the procedure outlined in Antolin \& Van Doorsselaere (2013), but the CHIANTI atomic data has not been used and an artificial spectral line is generated. Also, in Van Doorsselaere \& Nakariakov (2008) and Antolin \& Van Doorsselaere (2013), the intensity is taken to be proportional to the density squared (rather than linearly proportional in the current forward model) because, in these particular papers, coronal emission lines were studied.

Since spicules are optically thick, only the plasma up to an optical depth of $\tau=1$ at the front of the spicules is taken to contribute to the emission line. In this very simple model, we have mimicked this effect by integrating the emission from the cylinder only over the half facing the observer. We believe that the proper treatment of the optically thick emission would result in only minor changes.

The results of the computation are shown in Figure 9. We choose the same convention for the LOS angle as described in Figure 5, i.e., $\varphi_{0}$ is the angle between the LOS and the direction of the classic kink transverse motion. The top window shows the emission for the kink wave. In top window (bottom panel), we have $\varphi_{0}=0^{\circ}$, i.e., the LOS is along the direction of the classic kink transverse motion of the spicule. This is the same LOS as Special case I in Section 6.2.1, so it also results in a Doppler velocity profile, which is symmetric about the center of the spicule. The bright emission of the internal plasma (that is 10 times denser than the surrounding) is redshifted to $20 \mathrm{~km} \mathrm{~s}^{-1}$, because the classic kink transverse motion is aligned with the LOS. The counterstreaming motion of the plasma outside the cylinder results in blueshifted, fainter wings $(y \lesssim 800 \mathrm{~km}$ and $y \gtrsim 1200 \mathrm{~km}$ ). This is similar in appearance to the Doppler velocity profile shown in Figure 6(a), although the toy model in that particular case did not consider the external plasma. The
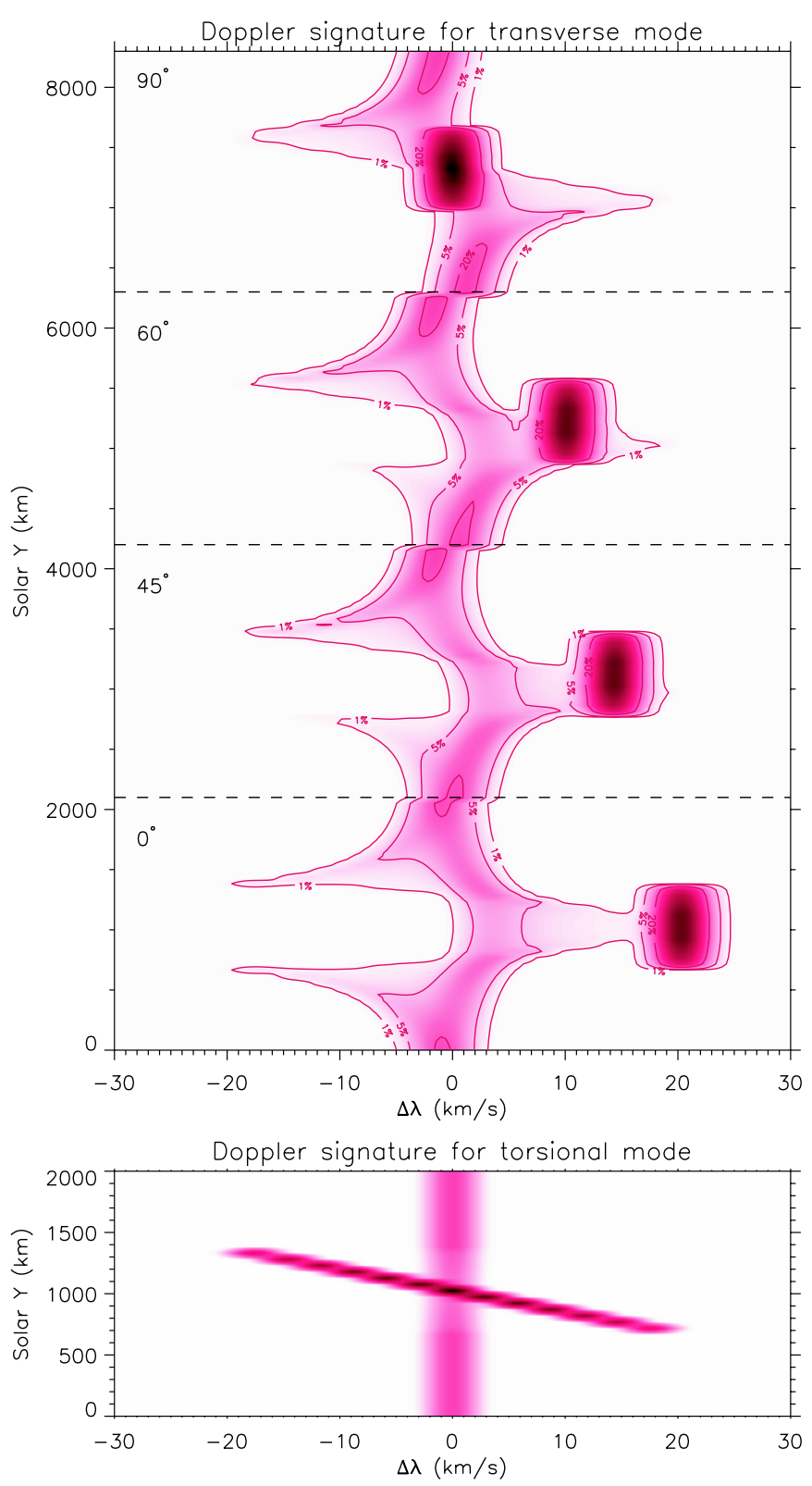

Figure 9. Integrated intensity of the transverse kink mode (top window) and the torsional mode (bottom window). In both windows, the intensity is shown as a function of the Doppler shift (horizontal axis) and the position across the cylinder (vertical axis). The color scale of light pink to dark pink indicates increasing emission, and white is no emission. The top window contains several panels corresponding to different viewing angles between the LOS and direction of classic kink transverse motion (indicated in the top left of each panel). The contour lines in the top window are shown for $1 \%, 5 \%$, and $20 \%$ of the maximum intensity when the LOS angle is $90^{\circ}$ relative to the direction of the classic kink motion.

(A color version of this figure is available in the online journal.)

reason for the change of Doppler sign from the center to the boundary of the tube in Figure $6(\mathrm{~d})$ was because $C_{\mathrm{ROT}}$ was chosen to be larger in magnitude and opposite in sign to $C_{\mathrm{TR}}$ at the tube boundary.

The top panel (top window) considers Special case II in Section 6.2.2, i.e., the LOS is perpendicular to the direction of the classic kink transverse motion $\left(\varphi_{0}=90^{\circ}\right)$. As expected, the emission from the cylinder is not Doppler shifted, because the velocity inside the cylinder has no component along the LOS. However, the counterstreaming motion around the cylinder is 


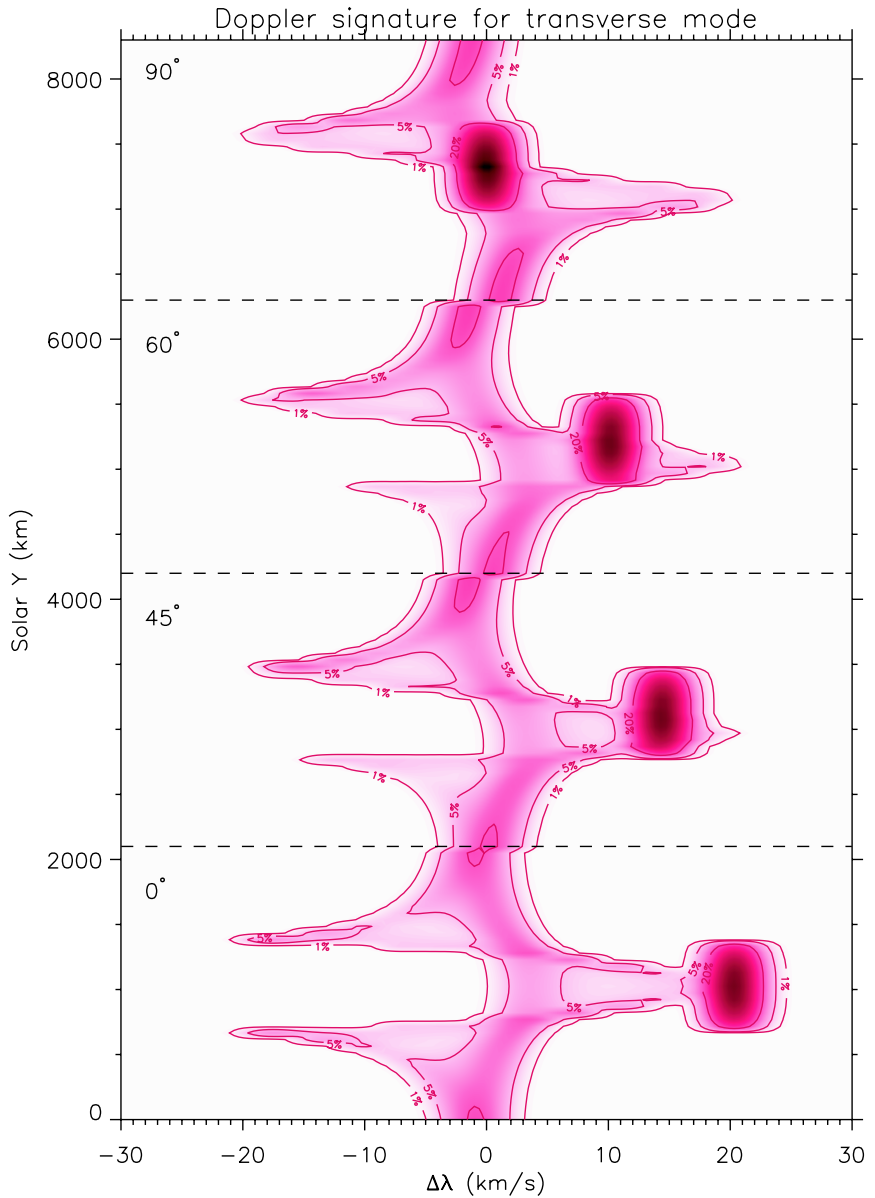

Figure 10. Same as Figure 9, but with a smooth density profile $l / R=1$.

(A color version of this figure is available in the online journal.)

still observed as an anti-symmetric blue and redshift close to the cylinder edge. $(y \lesssim 7000 \mathrm{~km}$ and $y \gtrsim 7500 \mathrm{~km})$. This is similar in appearance to the anti-symmetric Doppler velocity profile shown in Figure 7, but, again, the external plasma was not considered in that particular toy model. The reason for the antisymmetric Doppler velocity profile in Figure 7 was that $C_{\mathrm{ROT}}$ was chosen to be non-zero at the tube boundary. The cases for an angle of $\varphi_{0}=45^{\circ}$ and $\varphi_{0}=60^{\circ}$ are shown for intermediate angles where an asymmetric pattern is formed in the emission, see case of $\varphi_{0}=45^{\circ}$ with same LOS angle in Figure 8(d).

For comparison, the emission for the torsional Alfvén wave is shown in the lower window of Figure 9. To obtain this Doppler velocity profile, we have taken a static plasma outside the cylinder, and imposed a periodic solid body rotation inside the cylinder (with a maximum amplitude of $20 \mathrm{~km} \mathrm{~s}^{-1}$ at the edge of the cylinder). This would be representative for torsional Alfvén oscillations that are in phase at every radial position in the cylinder. The emission is characterized by a diagonal band in the wavelength-space domain, as shown in Equation (42) and illustrated in the bottom window of Figure 4. With this current, simple, forward model of the emission, the kink wave can produce a varying red/blue shift profile between the internal and external plasma that also changes with LOS angle. It can be seen, especially for an LOS angle of $\varphi_{0}=90^{\circ}$, the emission could be easily confused with the emission of a torsional Alfvén wave (see also Figure 7).

More realistically, we do not expect a plasma density discontinuity at the flux tube boundary, but a layer of plasma of some

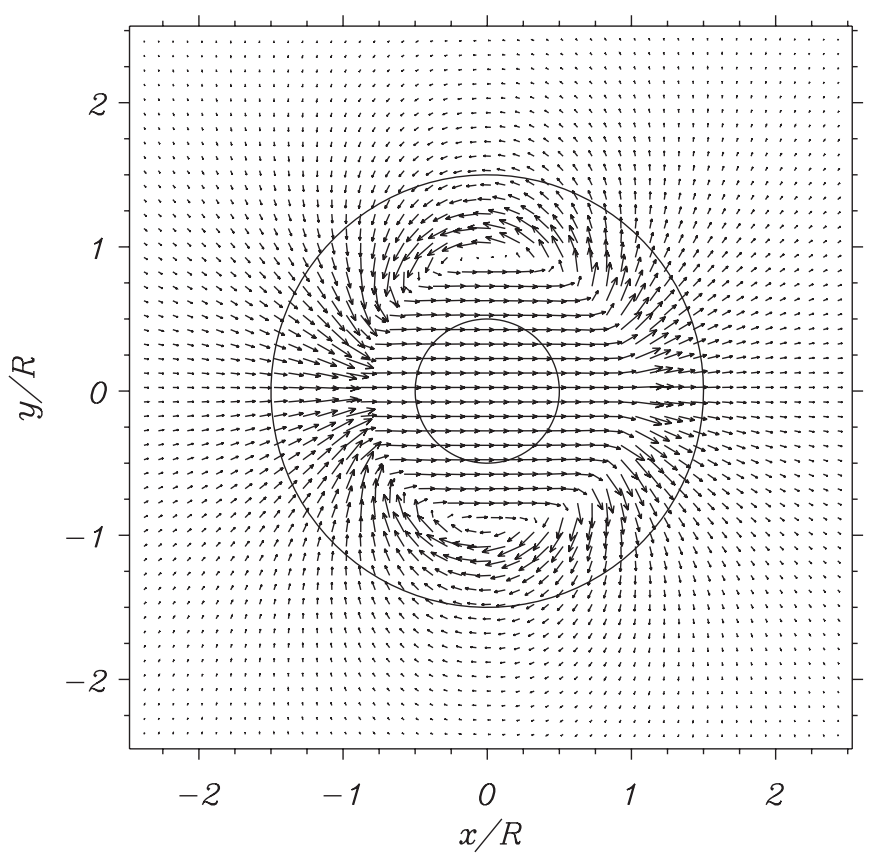

Figure 11. Kink "quasi-mode" displacement field (arrows) in a non-uniform tube with $l / R=1$. The annulus represents the non-uniform layer between the internal and external plasma.

finite thickness that changes in value from the internal to external densities, i.e., an inhomogeneous layer. In Figure 10, we do the same forward model with a smoothly changing density layer. To do so, the sharp boundary of the magnetic cylinder at $r=R$ is replaced by a smooth transitional layer of thickness $l$ that continuously connects the internal plasma density to the external plasma density. The non-uniform layer extends in the interval $[R-l / 2, R+l / 2]$. The integrated intensity and Doppler velocity from this configuration is equivalent to that expected from the kink "quasi-mode" shown in Figure 11 (with the same $l / R=1$ ratio). The physical origin of the "quasi-mode," which naturally arises in magnetic flux tubes which have a continuous variation in density between the internal and external plasma, is explained in the following section. Purely from the point of view of observed intensity, quite simply, the inhomogeneous layer would boost the strength of the Doppler velocity signal from the external velocity field. Compared with the top window in Figure 9, the blue-shifted wings (for the $\varphi_{0}=0^{\circ}$ case) in Figure (10) are more pronounced, and the intensity is more smoothed out over the symmetric Doppler velocity profile. Similarly, for the $\varphi_{0}=90^{\circ}$ case, the intensity is smoothed out over the diagonal created by the cylinder and the anti-symmetric wings. This generates a picture that is even more alike to the bottom window in Figure (9) for the torsional Alfvén wave, and would be even more difficult to distinguish observationally.

In De Pontieu et al. (2012), they forward-modeled the Doppler velocity produced by spicule motion based on a Monte Carlo approach. Although De Pontieu et al. only assumed the presence of $m=0$ rotational motion in their particular model, they did not discount the occurrence of $m>0$ values in observational data. In this section, we have highlighted where the Doppler signatures of $m=0$ and $m=1$ motion look similar or distinct depending on the LOS. Certainly, the most distinct signatures of the $m=1$ combined transverse and rotational motion should be searched for in the observational data, i.e., when the LOS is closely aligned with the classic kink transverse motion 
(e.g., bottom panel of top window in Figure 9 and bottom panel in Figure 10). In the next section, we consider the complexity in the time/space variation in the transverse and rotational components of a kink wave when a more realistic, inhomogeneous density layer is introduced between the internal and external plasma.

\section{MOTIONS IN A TRANSVERSELY NON-UNIFORM FLUX TUBE}

Certainly a magnetic flux tube with a piece-wise constant density is a crude approximation to the waveguides observed in the solar atmosphere. In this section, following on from the Doppler velocity forward model shown in Figure 10, which added a smoothly varying density layer between the internal and external plasma, we now investigate the effect of transverse density inhomogeneity on the kink wave displacement field in more detail. As in Section 7, the non-uniform layer of width $l$ extends in the interval $[R-l / 2, R+l / 2]$. A continuum of Alfvén frequencies appears due to the presence of the nonuniform layer. The kink mode frequency, which is necessarily between the internal and external Alfvén frequencies, lies now within the frequency continuum, and so the kink mode becomes resonantly coupled to Alfvén continuum modes. The kink mode energy is ideally transferred to the continuum modes and, as a consequence, the transverse motion linked to the kink mode is damped in time (see, e.g., Goossens et al. 2011).

As explained in Section 5, the kink mode has both radial and azimuthal components of the displacement, whereas the Alfvén continuum modes are essentially polarized in the azimuthal direction. As the energy from the kink mode is resonantly transferred to the Alfvén continuum modes, the flux tube motion changes from a purely transverse motion to a purely rotational motion in a timescale $\tau_{D}$ that depends on the properties of the equilibrium. This change of polarization was represented by the toy model in Section 5 by Equations (36) and (37).

A detailed study of the resonant absorption process beyond this simple but instructive toy model requires advanced analytic/ numerical MHD modeling. An attempt to incorporate the effect of resonant absorption from the analytic point of view is based on the assumption that a global kink normal mode still exists in the non-uniform case. In other words, it is possible to find in the non-uniform case a mode that is the direct descendant of the kink mode in a piece-wise uniform tube. The effect of resonant absorption would then be to add an imaginary part to the frequency of the kink mode, i.e., to introduce a damping timescale. Soler et al. (2013) used the method of Frobenius to express the perturbations in the non-uniform layer as a combination of a regular and singular series around the Alfvén resonance position (a similar approach was previously used by Hollweg 1990, in a Cartesian interface). The series expansion was used to connect through the non-uniform layer the perturbations in the internal plasma to those in the external plasma (see the full details of this procedure in Soler et al. 2013). This process allowed Soler et al. (2013) to find a dispersion relation for the kink mode in the non-uniform case. The study of the dispersion relation reveals that it is a multi-valued function and that there are no solutions on its principal Riemann sheet. The complex solution that can be related to the descendant of the kink mode in a piece-wise uniform tube is located on the next Riemann sheet. This result indicates that the kink mode in the non-uniform case is not a true, normal mode of the flux tube. Instead, the damping time of this kink "quasi-mode" has to be physically understood, not as a dissipation timescale, but as the timescale for which most of the energy of the flux tube global transverse motion is transferred to the azimuthally polarized continuum modes.

A simple mathematical expression for the transverse motion damping timescale can be derived assuming that the transverse inhomogeneity is confined to a layer much thinner than the radius of the tube, i.e., the thin boundary approximation or $l / R \ll 1$ (see, e.g., Hollweg \& Yang 1988; Ruderman \& Roberts 2002; Goossens et al. 2002). This condition is required to make the analysis mathematically simple although it may not be physically realistic for applications to actual solar atmospheric waveguides (Soler et al. 2014). In the thin boundary approximation, the kink "quasi-mode" damping timescale due to resonant absorption is

$$
\tau_{D}=F \frac{R}{l} \frac{\rho_{i}+\rho_{e}}{\rho_{i}-\rho_{e}} P,
$$

where $P$ is the kink mode period (the same as in a piece-wise uniform tube) and $F$ is a numerical factor that depends on the specific density profile considered in the non-uniform layer. This simple dependence on the density profile does not hold for thick transitional layers (Soler et al. 2014).

The displacement field of the kink "quasi-mode" in a nonuniform tube obtained by Soler et al. (2013) is plotted in Figure 11, which can be compared to that of the kink mode in a piece-wise uniform tube displayed in Figure 2. It is clear from Figure 11 that the motion in the non-uniform layer is mainly rotational due to the presence of the resonance. As happens for the interpretation of the damping timescale, special caution is needed when physically interpreting the LOS Doppler velocity profile that one would obtain from the displacement field displayed in Figure 11. The reason is that the "quasi-mode" primarily accounts for the global transverse and rotational motion generated by the kink wave, and does not describe the localized plasma motions linked to the Alfvén continuum modes. As time increases, more and more energy is transferred to the continuum modes and their contribution to the total displacement field becomes important when their amplitudes grow. Therefore, the displacement field of the "quasi-mode" may be interpreted as an approximation to the actual motion of the plasma at a sufficiently early stage of the oscillation only.

In order to better understand the polarization of the plasma motions as the oscillations progress through time, we have solved the full time-dependent problem. This allows us to advance without the inherent limitations of the approximate analytical modeling described in Sections 5-6. The linearized ideal MHD equations are numerically solved in a Cartesian coordinate system in the $x$ and $y$ directions (in $z$ direction we perform a Fourier analysis). We use the same numerical method as in Terradas et al. (2008). Since the aim here is to study the evolution of the flux tube displacement, we impose at $t=0$ an impulse in the $x$ direction mostly localized inside the tube $\left(0<\sqrt{x^{2}+y^{2}}<R+l / 2\right)$.

The evolution of the displacement field is plotted in Figure 12 at different times. In this plot the size of the arrows is proportional to the amplitude of the displacement. Since the loop is oscillating around the equilibrium position, the direction of the displacement field changes periodically with time and, more importantly, the spatial distribution of the displacement also changes. We clearly see how the transverse motions inside the tube decrease in amplitude as time increases while at the same time the azimuthal displacements inside the inhomogeneous layer keep growing. This is the mechanism of resonant 

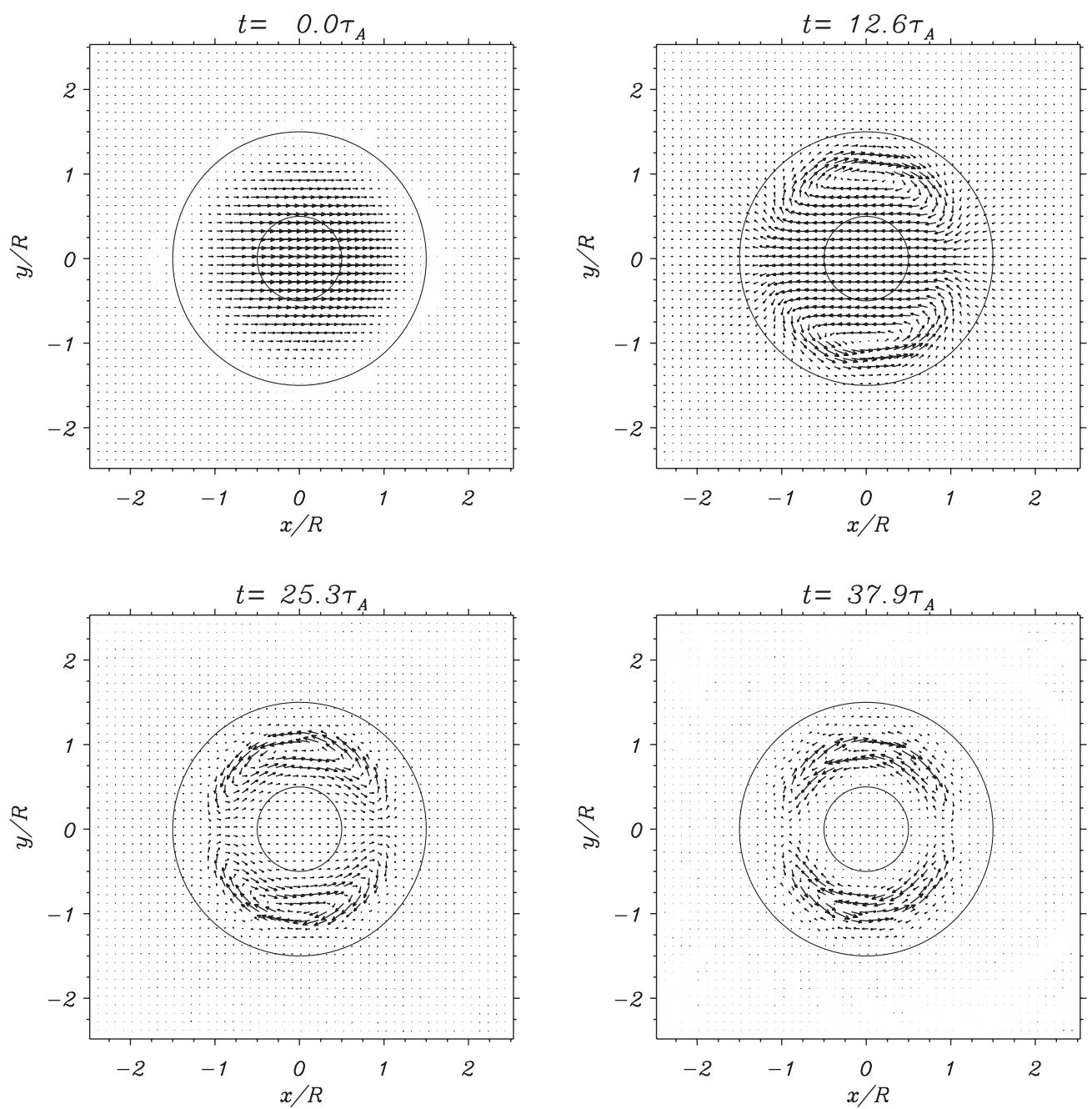

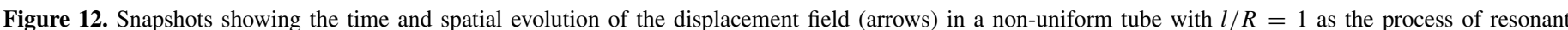

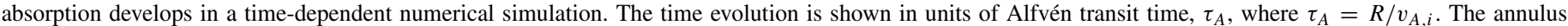
represents the non-uniform layer between the internal and external plasmas.

(An animation of this figure is available in the online journal.)

absorption and the final result is that all the energy becomes localized in anti-symmetric, azimuthal motions around the resonant layer, which is located at $r \approx R$ in our case. In essence, this is the process described using the simple toy model of Equations (36) and (37).

In connection to the accuracy of the "quasi-mode" modeling, now we can compare the "quasi-mode" displacement field of Figure 11 with the full numerical displacement field of Figure 12. The "quasi-mode" is a reasonably good approximation to the actual displacement field in the first few periods of the oscillation after the initial excitation. For instance, the "quasi-mode" displacement field qualitatively agrees with that shown in the upper right panel of Figure 12, which corresponds to the displacement field after almost two periods of the transverse oscillation. However, the displacement fields in the lower left and right panels of Figure 12, which roughly correspond to three and five periods of oscillation, respectively, are already significantly different from the "quasi-mode" displacement field. The plasma motions displayed in the lower left and right panels of Figure 12, especially those in the lower right panel, are mostly dominated by azimuthal motions in the non-uniform layer. These azimuthal motions localized in the non-uniform layer can be theoretically related to the contribu- tion of the Alfvén continuum modes, which is not captured by the "quasi-mode" approximation. In relation to actual Doppler velocity data, the relative amplitudes between the transverse and rotational velocities would depend on what stage of the mode conversion process was being observed. It would certainly be a great step forward to identify this process of mode conversion directly, e.g., in propagating kink waves by measuring the transfer of energy from transverse to rotational motion with height in the solar atmosphere.

\section{CONCLUSIONS}

It is hoped that this current work will go some way toward dispelling the widely held notion that only the axisymmetric torsional motion has rotational motion. It has been demonstrated that the velocity field of the kink wave is naturally a sum of both transverse and rotational motion, and due to its non-axisymmetric nature, its observed Doppler velocity profile varies with LOS. This is particularly timely since we now have modern, high temporal/spatial resolution spectroscopic instruments, e.g., TRIPPLE/SST, CRISP/SST, IBIS/DST, IRIS and the forthcoming ATST, that are capable of detecting variation of Doppler velocity trends across fine scale oscillating plasma structures such as chromospheric spicules/fibrils/mottels. 
We have shown that when the LOS is perpendicular to the direction of the classic kink transverse motion, the resultant Doppler velocity profile due to the presence of the kink wave's rotational motion can look very similar to that expected from a torsional Alfvén wave. Taking this fact into account is particularly important, e.g., to accurately interpret the varying Doppler velocity profiles across spicules in which ubiquitous transverse and rotational motions are observed to be present. We have also shown that the relative amplitudes of the transverse and rotational velocities in kink waves and their time/space evolution depend on the length scale of the transverse plasma density inhomogeneity and what stage of the mode conversion process is being observed. It is hoped that the latest high temporal/spatial resolution spectroscopic instruments will provide new and much sought after insight into this mode coupling, conversion, and time/space evolution as the waves propagate through the Sun's atmosphere.

M.G. acknowledges support from KU Leuven via GOA/ 2009-009 and also partial support from the Interuniversity Attraction Poles Programme initiated by the Belgian Science Policy Office (IAP P7/08 Charm). T.V.D. was sponsored by an Odysseus grant of the FWO Vlaanderen. M.G. and R.S. acknowledge the support from the Spanish MINECO and FEDER funds through project AYA2011-22846. R.S. also acknowledges support from CAIB through the "grups competitius" scheme and FEDER Funds and the Spanish Ministry of Economy and Competitiveness through a Juan de la Cierva grant, from the Ministry of Education, Culture, and Sport through project CEF11-0012, and from the UIB Vicerectorat dInvestigaci i Postgrau. J.T. acknowledges support from the Spanish Ministerio de Educación y Ciencia through a Ramón y Cajal grant and financial support from MICINN/MINECO and FEDER Funds through grant AYA2011-22846. J.T. also acknowledges funding from CAIB through the Grups Competitius scheme and FEDER Funds. G.V. acknowledges the support of the Leverhulme Trust (UK).

\section{REFERENCES}

Antolin, P., \& Van Doorsselaere, T. 2013, A\&A, 555, 74

Appert, K., Gruber, K., \& Vaclavik, J. 1974, PhFl, 17, 1471
Beckers, J. M. 1968, SoPh, 3, 367

Bjølseth, S. 2008, Masters thesis, Univ. Oslo

Cally, P. 1985, AuJPh, 38, 825

De Pontieu, B., Carlsson, M., Rouppe van der Voort, L. H. M., et al. 2012, ApJL, 752, L12

Edwin, P. M., \& Roberts, B. 1983, SoPh, 88, 179

Goossens, M. 2008, in IAU Symp. 247, Waves \& Oscillations in the Solar Atmosphere: Heating \& Magneto-Seismology, ed. R. Erdélyi \& C. A Mendoza-Briceño (Cambridge: Cambridge Univ. Press), 228

Goossens, M., Andries, J., \& Aschwanden, M. J. 2002, A\&A, 394, L39

Goossens, M., Andries, J., Soler, R., et al. 2012, ApJ, 753, 111

Goossens, M., Erdélyi, R., \& Ruderman, M. R. 2011, SSRv, 158, 289

Goossens, M., Hollweg, J. V., \& Sakurai, T. 1992, SoPh, 138, 233

Goossens, M., Ruderman, M. S., \& Hollweg, J. V. 1995, SoPh, 157, 75

Goossens, M., Terradas, J., Andries, J., Arregui, I., \& Ballester, J. L. 2009, A\&A, 503,213

He, J.-S., Marsch, E. Tu, C.-Y., \& Tian, H. 2009a, ApJL, 705, L217

He, J.-S., Tu, C.-Y., Marsch, E., et al. 2009b, A\&A, 497, 525

Hollweg, J. V. 1990, JGR, 95, 2319

Hollweg, J. V., \& Yang, G. 1988, JGR, 93, 5423

Kuridze, D., Morton, R. J., Erdélyi, R., et al. 2012, ApJ, 750, 51

Kuridze, D., Verth, G., Mathioudakis, M., et al. 2013, ApJ, 779, 82

Lin, Y., Soler, R., Engvold, O., et al. 2009, ApJ, 704, 870

Makita, M. 2003, PNAOJ, 7, 1

McIntosh, S. W., \& De Pontieu, B. 2012, ApJ, 761, 138

Morton, R. J., Verth, G., Fedun, V., Shelyag, S., \& Erdélyi, R. 2013, ApJ, 768,17

Morton, R. J., Verth, G., Hillier, A., \& Erdélyi, R. 2014, ApJ, 784, 29

Morton, R. J., Verth, G., Jess, D. B., et al. 2012, NatCo, 3, 1315

Okamoto, T. J., \& De Pontieu, B. 2011, ApJL, 736, L24

Ruderman, M. S., \& Roberts, B. 2002, ApJ, 577, 475

Sakurai, T., Goossens, M., \& Hollweg, J. V. 1991, SoPh, 133, 227

Soler, R., Goossens, M., Terradas, J., \& Oliver, R. 2013, ApJ, 777, 158

Soler, R., Goossens, M., Terradas, J., \& Oliver, R. 2014, ApJ, 781, 111

Spruit, H. C. 1982, SoPh, 75, 3

Terradas, J., Arregui, I., Oliver, R., et al. 2008, ApJ, 679, 1611

Terradas, J., Goossens, M., \& Verth, G. 2008, A\&A, 524, 23

Van Doorsselaere, T., Andries, J., Poedts, S., \& Goossens, M. 2004, ApJ, 606, 1223

Van Doorsselaere, T., \& Nakariakov, V. A. 2008, in ASP Conf. Ser. 397, First Results From Hinode, ed. S. A. Matthews, J. M. Davis, \& L. K. Harra (San Francisco, CA: ASP), 58

Verth, G., Goossens, M., \& He, J.-S. 2011, ApJL, 733, L15

Verth, G., Terradas, J., \& Goossens, M. 2010, ApJL, 718, L102

Wentzel, D. G. 1979, A\&A, 76, 20

Wilson, P. R. 1979, A\&A, 71, 9

Wilson, P. R. 1980, A\&A, 87, 121 